\title{
Quantum ballistic and adiabatic electron transport studied with quantum point contacts
}

\author{
B J van Wees, L P Kouwenhoven, E M M Willems, C J P M Harmans, and J E Moouj \\ Faculty of Applied Physics, Delft University of Technology, 2600 GA Delft, The Netherlands \\ H van Houten, C W J Beenakker, and J G Willamson \\ Philips Research Laboratories, $5600 \mathrm{JA}$ Eindhoven, The Netherlands \\ C $\mathrm{T}$ Foxon \\ Philips Research Laboratories, Redhill, Surrey RH1 5HA, United Kingdom
}

(Received 26 October 1989, revised manuscript received 20 December 1990)

\begin{abstract}
We present an experimental and theoretical study of quantum ballistic transport in single quantum point contacts (QPC's), defined in the two-dimensional electron gas (2DEG) of a high-mobility GaAs $/ \mathrm{Al}_{033} \mathrm{Ga}_{06}{ }_{67} \mathrm{As}$ heterojunction In zero magnetic field the conductance of quantum point con tacts shows the formation of quantized plateaus at multıples of $2 e^{2} / h$ The experimental results are explaned with a simple model Deviations from ideal quantization are discussed The experimental results are compared with model calculations Energy averaging of the conductance has been studied, both as a function of temperature and voltage across the device The application of a magnetic field leads to the magnetic depopulation of the one dimensional subbands in the QPC It is shown that the zero field quantization and quantization in high magnetic fields are two limiting cases of a more general quantization phenomenon We use quantum point contacts to study the highmagnetic-field transport in a $2 \mathrm{DEG}$ Quantum point contacts are used to selectively populate and detect edge channels The experments show that scattering between adjacent edge channels can be very weak, under certain circumstances even on length scales longer than $200 \mu \mathrm{m}$ This adiabatic transport has resulted in the observation of an anomalous integer quantum Hall effect, in which the quantization of the Hall conductance is not determined by the number of Landau levels in the bulk 2DEG, but by the number of Landau levels in the QPC's instead Related effects are the anomalous quantization of the longitudinal resistance and the adiabatic transport through QPC's in series A theoretical description for transport in the presence of Shubnikov-de Haas $(\mathrm{SdH})$ backscattering is given This model explans the experimentally observed suppression of the $\mathrm{SdH}$ oscillations due to the selective population or detection of edge channels Finally, we demonstrate that the combina tıon of a QPC and a bulk Ohmic contact can act as a controllable edge-channel mixer
\end{abstract}

\section{INTRODUCTION}

The fundamental pioperties of election transport are best studied in the ballistic : egime In this regime the elastic and inelastic mean free paths $l_{c}$ and $l_{t}$ are both larger than the dimensions of the conductor through which the electrons travel The motion of the electrons is then completely determined by the (smooth) electiostatic potentral, which defines the conductor, and is not disturbed by interactions with phonons, impuities, etc A classical description of ballistic transport suffices when the dimensions of the conductor are large compared to the Fermi wavelength $\lambda_{I}$ of the electrons When the device dimensions become comparable to $\lambda_{I}$, the quantum ballistic regime is entered In this regime the wavelike nature of the electrons becomes prominent

The two-dimensional electron gas (2DEG) of a highmobility $\mathrm{GaAs} / \mathrm{Al}_{0}{ }_{33} \mathrm{Ga}_{0}{ }_{67} \mathrm{As}$ heterojunction is a very attractive system for the study of quantum ballistic transport At low temperatures both $l_{e}$ and $l$, can become relattvely large $(>10 \mu \mathrm{m})$ Also, $\lambda_{F}$ is relatively large (typically $40 \mathrm{~nm}$ ) With modern microfabication techniques it is therefore possible to fabricate devices in a 2 DEG that operate in the quantum ballistic regime We have employed a split-gate technique ${ }^{12}$ to fabricate quantum point contacts (QPC's) These QPC's are short and narrow constrictions, with dimensions comparable to $\lambda_{F}$ An attractive feature of the split-gate technique is that the properties of the QPC's can be controlled continuously by the applied gate voltage This has enabled us to perform a detailed study of the quantum ballistic transpoit regime ${ }^{3}$

This paper consists of two major parts (Secs III and IV) After the description of the device layout and the experimental setup in Sec II, we study the ballistic transport through single QPC's in Sec III Section III A gives a brief introduction of quantum ballistic transport The experiments that reveal the quantization of the ballistic conductance of quantum point contacts in the absence of a magnetic field are presented in Sec III B The results will be explained with a simple model Deviations from ideal quantization are discussed in Sec IIIC In Sec III D we study the influence of energy averaging due to a finite temperature and finite voltage across the QPC's A comparison of our results with model calculations will be given in Sec III $\mathrm{E}$ The application of a perpendicular magnetic field leads to the magnetic depopulation of the 
one-dimensional subbands in the QPC. The quantization is preserved, and it is shown that the zero-field quantization and the quantum Hall effect (QHE) in a QPC are two limiting cases of a more general quantization phenomenon (Sec. III F). The conclusion of this section is in Sec. III G.

In Sec. IV we present a detailed theoretical and experimental investigation of high-magnetic-field transport in a 2DEG, studied with QPC's. Recently a simple and appealing model for electron transport in the quantum Hall regime ${ }^{4,5}$ has been proposed. ${ }^{6-8}$ The main ingredients of this model are the so-called edge channels. These edge channels consist of the current-carrying electron states of each Landau level, and are located at the boundaries of the 2DEG. We will give a brief description of this model in Sec. IV A. The power of the quantum point technique is that the transmission properties of QPC's can be controlled by the applied gate voltage. The most important property of QPC's in high magnetic fields is that they, when used as current probes, can selectively inject current into specific edge channels. When used as voltage probes they can selectively measure the occupation of specific edge channels. A description of the highmagnetic-field transport in single QPC's is given in Sec. IV B.

The selective properties of the QPC's allow us to perform a detailed study of the role of contacts in the QHE. An important result of our investigation is that scattering between adjacent edge channels (located at the same 2DEG boundary) can be very weak in high magnetic fields, which implies that adiabatic transport can take place. Electrons travel through the $2 \mathrm{DEG}$ with conservation of their quantized magnetic energy (Landau-level index), with only a little chance of being scattered into other edge channels. The combination of this quantum adiabatic transport with the selective population and detection of edge channels by QPC's has resulted in the observation of an anomalous integer QHE (Ref. 9) (Sec. IV C). The quantization of the Hall conductance is not determined by the number of Landau levels in the bulk 2DEG, but by the number of Landau levels in the QPC's instead. Related phenomena are the anomalous quantization of the longitudinal resistance and the quantum adiabatic transport in QPC's in series (Sec. IV D).

Next we used QPC's to perform a detailed study of the scattering between edge channels. In Sec. IV $\mathrm{E}$ we give a description of the scattering processes in the $2 \mathrm{DEG}$. We make a distinction between intra-Landau-level scattering (scattering between edge channels belonging to the same Landau level) and inter-Landau-level scattering (scattering between edge channels belonging to different Landau levels). In our model the Shubnikov-de Haas (SdH) oscillations arise from backscattering of electrons in the upper (highest occupied) Landau level. The experiments that show that the $\mathrm{SdH}$ oscillations can be suppressed, either by selective population or by selective detection of edge channels, are presented in Sec. IV F. These results show that under certain circumstances the scattering between adjacent edge channels can be weak even on a macroscopic $(>200-\mu \mathrm{m})$ length scale. ${ }^{10}$ Another illustration of the nonlocal transport is given in Sec. IV G, where we demonstrate that the voltage measured with a particular voltage probe can be strongly affected by the transmission properties of an adjacent voltage probe. This shows that a voltage contact that consists of a QPC and an Ohmic contact can act as a controllable "edge-channel mixer." Section IV $\mathrm{H}$ concludes the paper.

The main body of our results has been published in earlier papers. ${ }^{9-12}$

\section{DEVICE LAYOUT AND EXPERIMENTAL SETUP}

In Fig. 1 we show the schematic layout and a micrograph of the devices. Identical devices have been used for the study of coherent electron focusing, ${ }^{13-15}$ hot electron focusing, ${ }^{16}$ nonlinear transport in QPC's, ${ }^{17}$ and the Aharonov-Bohm effect in singly connected point contacts. ${ }^{18}$ The starting material is a nigh-mobility twodimensional electron gas, which is present in a $\mathrm{GaAs} / \mathrm{Al}_{0.33} \mathrm{Ga}_{0.67} \mathrm{As}$ heterojunction, grown by molecular-beam-epitaxy (MBE) techniques. The structure consists of a 4- $\mu \mathrm{m}$ GaAs layer (grown on semiinsulating GaAs), followed by a 20-nm undoped $\mathrm{Al}_{0.33} \mathrm{Ga}_{0.67} \mathrm{As}$ spacer layer, a $40-\mathrm{nm}$ doped $\left(1.33 \times 10^{18}\right.$ $\left.\mathrm{cm}^{-3} \mathrm{Si}\right) \mathrm{Al}_{0.33} \mathrm{Ga}_{0.67} \mathrm{As}$ layer, and a $20-\mathrm{nm}$ undoped

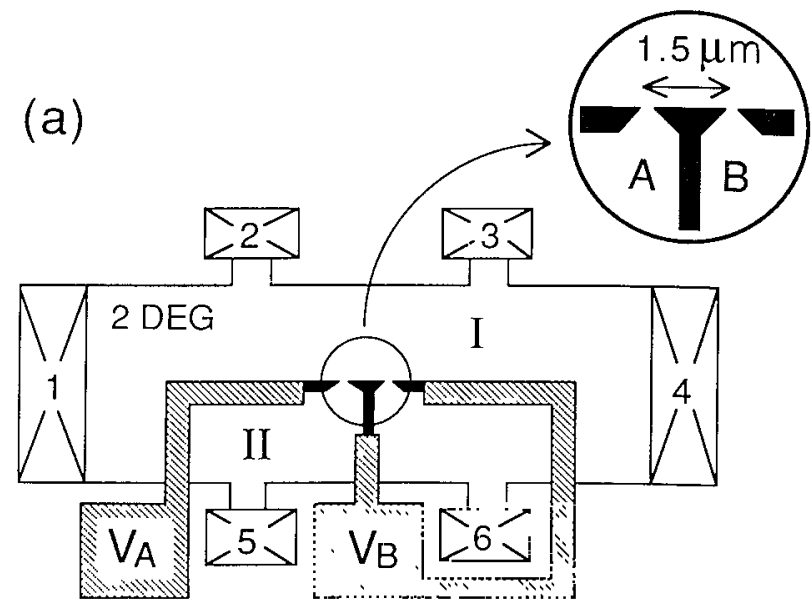

(b)

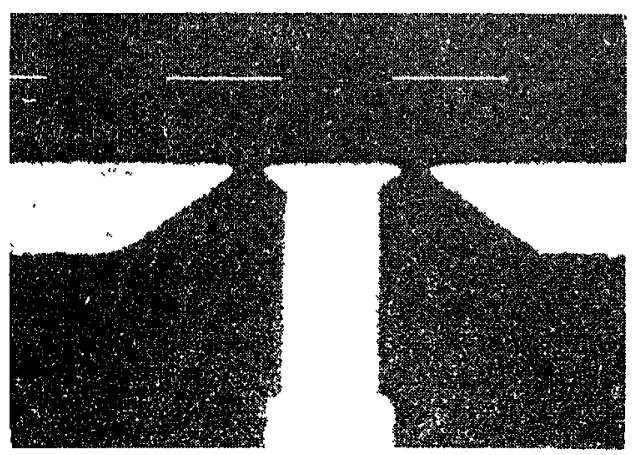

FIG. 1. (a) Schematic layout of the device. The gates define two adjacent quantum point contacts $A$ and $B$. (b) Micrograph, showing the gate on top of the heterojunction, which defines two adjacent QPC's. The white bar is $1 \mu \mathrm{m}$. 
GaAs cap layer. The electron density of the $2 \mathrm{DEG}$ is $3.6 \times 10^{15} / \mathrm{m}^{2}$, which results in a Fermi energy $E_{F} \approx 12$ $\mathrm{meV}$, and a Fermi wavelength $\lambda_{F} \approx 40 \mathrm{~nm}$. The elastic mean free path (at $4.2 \mathrm{~K}$ ) is $9 \mu \mathrm{m}$ (the mobility is 85 $\left.\mathrm{m}^{2} / \mathrm{V} \mathrm{s}\right)$. Ohmic bulk contacts $1-6$ are fabricated by alloying $\mathrm{Au} / \mathrm{Ge} / \mathrm{Ni}$. A Hall bar $(200 \mu \mathrm{m}$ wide and $600 \mu \mathrm{m}$ long) is defined by optical lithography and wet chemical mesa etching. Gates $A$ and $B(20-\mathrm{nm} \mathrm{Au})$ are fabricated by a combination of optical lithography (hatched section), electron lithography (solid section), and lift off techniques.

The QPC's are defined by a split-gate technique, which was pioneered by Thornton et al. ${ }^{1}$ and Zheng et $a l .^{2}$ for the study of low-dimensional electron transport. ${ }^{19}$ An attractive feature of this technique is that contact with the $2 \mathrm{DEG}$, which is located about $60 \mathrm{~nm}$ below the surface, is avoided during the fabrication process. This prevents a possible reduction of the electron mobility due to surface damage. Application of a negative gate voltage $V_{g}=-0.6 \mathrm{~V}$ depletes the electron gas underneath the gate. As a result, two quantum point contacts $A$ and $B$ are defined, with a lithographic width of $250 \mathrm{~nm}$ and a separation of $1.5 \mu \mathrm{m}$. A further reduction of the gate voltage creates a saddle-shaped potential at the QPC's, and reduces their width and electron density. The QPC's are completely pinched off at $\approx-2.2 \mathrm{~V}$. The two separate gates make it possible to control the QPC's individually. As can be seen in Fig. 1, QPC $B$ is controlled by the gate voltage $V_{B}$, whereas QPC $A$ is controlled by both $V_{A}$ and $V_{B}$. It was found experimentally that the properties of QPC $A$ are approximately determined by the effective gate voltage $\left(V_{A}+V_{B}\right) / 2$.

We have investigated several nominally identical samples. In Sec. III we present experimental results of sample 1. Thermal cycling between room temperature and liquid-helium temperature resulted in a gradual deterioration of the quality of the quantization in zero magnetic field in this sample. Therefore the conductance of this sample obtained in different measurement runs shows a different quality of quantization, as well as different fine structure. However, the overall behavior of the sample did not change. In Sec. IV results on sample 2 are presented. The results obtained from these samples are typical for the remainder of the investigated samples.

The experiments were performed either in a pumped ${ }^{4} \mathrm{He}$ cryostat or in a ${ }^{3} \mathrm{He}^{4}{ }^{4} \mathrm{He}$ dilution refrigerator. The measurement leads were filtered to prevent rf interference. A phase-sensitive lock-in technique was used, with the voltages across the device kept below $k T / e$ to prevent energy averaging of the conductance.

\section{QUANTUM BALLISTIC TRANSPORT AND QUANTIZED CONDUCTANCE IN SINGLE QUANTUM POINT CONTACTS}

\section{A. Ballistic transport through quantum point contacts}

An important feature of ballistic transport is its nonlocality. The electron distribution (both in energy and momentum space) in a given section of the conductor is determined by scattering processes that have occurred in other sections of the conductor. This is the reason that a description of electron transport in which a local electric field is the driving agent is not suitable for the description of ballistic transport. Instead, a global description has to be given, in which current flows as a result of the difference in electrochemical potentials between different parts of the conductor. The electrochemical potential $\mu$ indicates up to which energy (kinetic plus electrostatic) the electronic states are occupied. A net current flows when the electron states that carry current in one direction are occupied up to a different energy than the electron states that carry current in the opposite direction. In this description of electron transport the resistance is caused by the backscattering of electrons. Landauer ${ }^{20}$ has proposed that resistance can be described with transmission and reflection probabilities, which indicate the fraction of the current that is transmitted or reflected by an obstacle. In the diffusive regime, where the mean free path between collisions with impurities is smaller than the dimensions of the conductor, the backscattering results from these impurity collisions. In the ballistic regime the backscattering is caused by the boundaries of the conductor itself.

The most elementary device to study ballistic transport is a so-called point contact. A point contact, first proposed by Sharvin, ${ }^{21}$ basically consists of a narrow and short constriction that connects two wider conductors. ${ }^{22}$ Both its width and length are less than the elastic and inelastic mean free paths. The description of the electron transport is as follows: The two wide conductors on either side of the constriction act as electron reservoirs that emit and absorb electrons. A voltage difference $V$ that is applied between the two regions creates a difference in electrochemical potential $e V=\mu_{L}-\mu_{R}$. As a result, electrons will impinge on the point contact from the right with energies up to $\mu_{R}$ and from the left with energies up to $\mu_{L}$. The net current $I$ through the point contact is therefore determined by the transmission probability of electrons in the energy interval between $\mu_{R}$ and $\mu_{L}$. When the applied voltage is low enough $\left(e V \ll E_{F}\right)$, the two-terminal conductance $G_{c}$ of the point is given by the Landauer formula

$$
G_{c}\left(E_{F}\right)=\frac{e I}{\mu_{L}-\mu_{R}}=\frac{2 e^{2}}{h} T\left(E_{F}\right),
$$

with $T\left(E_{F}\right)$ the transmission probability at the Fermi energy, and in which we have introduced the conductance quantum $2 e^{2} / h$. The ballistic point-contact resistance is exclusively determined by elastic processes. Dissipative processes in the wide reservoirs will equilibrate the electron distribution. In the ballistic regime these processes occur sufficiently far away from the point contact, and do not influence the resistance.

In metals the Fermi wavelength is typically a few angstroms, and is usually much smaller than the width of the point contact. This means that the transmission probability $T\left(E_{F}\right)$ can be evaluated classically, and the point-contact conductance is expected to be proportional to its width. In the experiments described in the following section we will measure the conductance of a quan- 
tum point contact as a function of its width. The fact that the width ( $250 \mathrm{~nm}$ or less) is comparable to $\lambda_{F}(\approx 40 \mathrm{~nm})$ yields a result that is stikingly different from the classical result.

\section{B. Conductance quantization in a quantum point contact}

The resistance of QPC $A$ is measured in zero magnetic field as a function of applied gate voltage $V_{A}=V_{B}$ at 0.6 K. A three-terminal setup is used, with voltage contacts 1 and 5 and current contacts 4 and 5 (see Fig. 1). ${ }^{23}$ Figure 2 shows the conductance $G_{c}$, which was obtained from the measured resistance after subtraction of a constant series resistance of $400 \Omega$. This resistance was chosen to match the plateaus with their corresponding quantized values, and is in reasonable agreement with the estimated series resistance, based on the sheet resistance of the $2 \mathrm{DEG}(\approx 20 \Omega)$ and the geometry $(\approx 16$ squares $)$ of region II.

The conductance of the QPC shows a sequence of quantized plateaus $^{11}$ at multiples of $2 e^{2} / h$. In the gatevoltage interval between the formation of the QPC at $-0.6 \mathrm{~V}$ to pinch off at $-2.2 \mathrm{~V}, 16$ plateaus are observed. A close examination of Fig. 2 shows that several plateaus are quite flat, whereas others show some fine structure. Similar results have been obtained by Wharam et al., who discovered the conductance quantization in short $(\approx 0.6 \mu \mathrm{m})$ and narrow channels, also defined with a split-gate technique. ${ }^{24,25}$

We have studied several nominally identical QPC's. They all show the steplike structure in $G_{c}\left(V_{g}\right)$. However, the fine structure in between the plateaus is different for each device. Also some devices show structure on the plateaus themselves. In our device geometry it is difficult to determine the accuracy of the quantization at the plateaus, because the series resistance may depend slightly on the applied gate voltage. ${ }^{26}$ However, a prerequisite for accurate quantization is that the plateaus are flat, and do not show fine structure. The results, therefore, show that the quantization is not exact. ${ }^{27}$ We will discuss the devia-

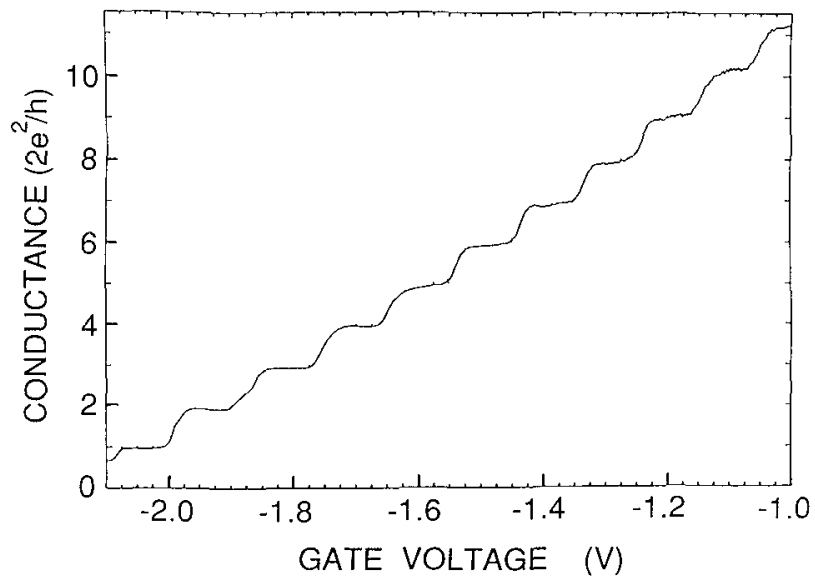

FIG. 2. Quantized conductance of a quantum point contact at $0.6 \mathrm{~K}$. The conductance was obtained from the measured resistance after subtraction of a constant series resistance of 400 $\Omega$. tions from exact quantization in detail in Secs. III C and III E.

The explanation for the observed conductance quantization is very elementary. We assume that we can model the QPC as a channel with finite length, in which the electrons are confined laterally by a parabolic potential $\frac{1}{2} m^{*} \omega_{0}^{2} x^{2}$, in which $m^{*}=0.067 m_{0}$ is the effective mass of the electrons, and $\omega_{0}$ indicates the strength of the lateral confinement. This choice of confinement is not essential for the result, but is a realistic approximation when the QPC's are near pinch off. ${ }^{28}$ The lateral confinement leads to the quantization of the lateral motion, and the formation of one-dimensional subbands. We obtain the following dispersion relation for the electron states in the QPC:

$$
E_{n}\left(k_{y}\right)=\left(n-\frac{1}{2}\right) \hbar \omega_{0}+\frac{\hbar^{2} k_{y}^{2}}{2 m^{*}}+e V_{0},
$$

which is the sum of the quantized lateral motion ( $n=1,2, \ldots$ is the index of the 1D subbands), the kinetic energy along the channel ( $k_{y}$ is the wave number for the motion along the channel), and the electrostatic energy $e V_{0}$ in the QPC. Figure 3 shows the occupied electron states at two different gate voltages. The analysis of the magnetoresistance of the QPC's in Sec. III F shows that the effect of the gate voltage is twofold: A more negative gate voltage increases the confinement and thus the energy separation $\hbar \omega_{0}$. As a second effect the electrostatic potential potential $V_{0}$ in the QPC is raised. As can be seen in Fig. 3, both effects reduce the number of occupied subbands $N_{c}$.

For the evaluation of the conductance $G_{c}$ we assume that all electron states with positive velocity $v_{y}=(1 / \hbar)\left[d E_{n}\left(k_{y}\right) / d k_{y}\right]$ are occupied to $\mu_{L}$ and all electron states with negative $v_{y}$ are occupied to $\mu_{R}$. This is equivalent to the assumption that no reflection occurs at both ends of the channel. Furthermore, we assume that the channel is long enough to prevent a contribution of evanescent waves to the conductance. The expression for $G_{c}$ now reads

$$
G_{c}=\frac{1}{V} \sum_{n=1}^{N_{c}} \int_{\mu_{R}}^{\mu_{L}} \frac{1}{2} e N_{n}(E) v_{n}(E) d E .
$$

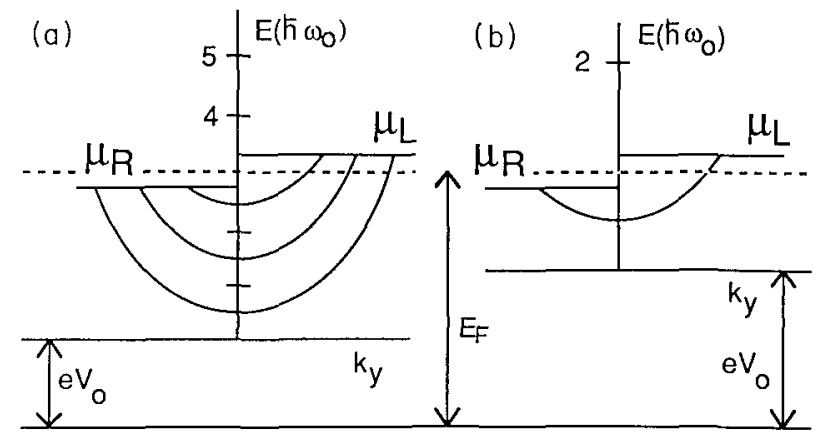

FIG. 3. Occupied electron states in the channel at two different gate voltages in the case of a current flow through the channel. In equilibrium the electron states are occupied up to the bulk Fermi energy $E_{F}$. An applied voltage creates a difference $e V=\mu_{R}-\mu_{L}$ between the electrochemical potentials of the reservoirs. 
The product of the 1D density of states (including both spin orientations) $N_{n}(E)=2 / \pi\left[d E_{n}\left(k_{y}\right) / d k_{y}\right]^{-1}$ and the group velocity $v_{n}(E)=(1 / \hbar)\left[d E_{n}\left(k_{y}\right) / d k_{y}\right]$ is energy independent, and equal to $4 / h$. This is an important feature of $1 \mathrm{D}$ transport and gives the result

$$
G_{c}=\frac{2 e^{2}}{h} N_{c} \text { with } N_{c}=\text { int }\left(\frac{E_{F}-e V_{0}}{\hbar \omega_{0}}+\frac{1}{2}\right),
$$

in which int denotes the truncation to an integer. The conductance is simply given by the conductance quantum $2 e^{2} / h$, multiplied by the number of occupied subbands in the QPC. Prior to the experimental discovery of the quantized point-contact conductance, the possibility of a quantized contact resistance between two reservoirs was anticipated by Imry. ${ }^{29}$ However, it was not expected at that time that an experimental system would show conductance quantization in such a clear and convincing way.

It can be shown that a classical evaluation of the point-contact conductance gives the result $G_{c}=\left(2 e^{2} / h\right)\left(E_{F}-e V_{0}\right) / \hbar \omega_{0}$. A comparison with Eq. (4) shows that the difference between classical and quantum results does not exceed $2 e^{2} / h$. This shows that in the limit of $G_{c} \gg 2 e^{2} / h$ the difference between quantum and classical results becomes unimportant.

\section{Deviations from ideal quantization}

Although the model of a channel with a finite length is clearly oversimplified, we can nevertheless use it to explain some of the features of the data. In this section we focus on the transition regions in between the quantized plateaus. We will explain the absence of quantization in these regions by the (partial) reflection of electron waves at both ends of the channel. A sudden widening of the channel, or change in electrostatic potential, at both ends of the channel will induce a partial reflection of the electron waves. This can be compared with the reflection of waves at an open-ended waveguide. In a first-order approximation the electron waves in a particular subband (or waveguide mode) are reflected in the same subband. We can then define a reflection probability $R$, which describes the fraction of the current carried by a subband that is reflected at the ends of the channel. In a onedimensional model the reflection probability for an abrupt potential step is given by

$$
R=\left|\frac{k_{y 1}-k_{y 2}}{k_{y 1}+k_{y 2}}\right|^{2},
$$

in which $k_{y 1}$ and $k_{y 2}$ are the longitudinal wave numbers inside and outside the channel. The transition regions between the quantized plateaus can now be understood with Eq. (5). The threshold for transmission of the $n$th subband is given by $E_{F}=e V_{0}+\left(n-\frac{1}{2}\right) \hbar \omega_{0}$. Slightly above the threshold, $k_{y 1}=\left\{2 m^{*}\left[E_{F}-e V_{0}-\left(n-\frac{1}{2}\right) \hbar \omega_{0}\right] /\right.$ $\left.\hbar^{2}\right\}^{1 / 2}$ is very small, and Eq. (5) shows that $R$ is near unity. The $n$th subband does not yet contribute significantly to the conductance. When $e V_{0}+\left(n-\frac{1}{2}\right) \hbar \omega_{0}$ is reduced further by increasing the gate voltage, $k_{y 1}$ increases, $R$ slowly drops to zero, and the conductance gradually reaches its quantized value.

Due to the possibility of multiple reflections at both ends of the channel, we also expect to observe transmission resonances. ${ }^{30}$ When we assume equal reffection probabilities $R$ at both ends of the channel we can write the conductance of the QPC as

$$
G_{c}=\frac{2 e^{2}}{h}\left(N+\frac{(1-R)^{2}}{1-2 R \cos \left(2 k_{y 1} L\right)+R^{2}}\right),
$$

in which $L$ is the length of the channel. This equation expresses that $G_{c}$ can be written as the sum of the quantized conductance of $N$ low-lying subbands (with low quantum number $n$ ) and the (resonant) transmission of the upper (highest occupied) subband. Equation (6) predicts transmission resonances in the transition regions between the quantized plateaus, where $R \neq 0$. An important feature of Eq. (6) is that even in the case of a finite reflection probability $R$, the conductance can still be quantized, provided that the condition for resonant transmission is satisfied: $2 k_{y 1} L=$ integer $\times 2 \pi$.

Figures 4 and 5 (upper traces) show experimental results. The data illustrate the transition from the first to the second plateau. Three maxima and two minima are observed, of which the second and the third maximum approach the quantized value $4 e^{2} / h$. The fact that the first maximum does not reach the quantized value may be due to the unequal reflection probabilities at both ends of the channel [note that the geometry of the QPC's is not symmetric (see Fig. 1)]. The number of observed resonances allows us to make an estimate of the length $L$ of the channel. At the threshold for the transmission of the third subband, the longitudinal wave number of the second subband is given by $k_{y 1}=\left(2 m^{*} E / \hbar^{2}\right)^{1 / 2}$, with the

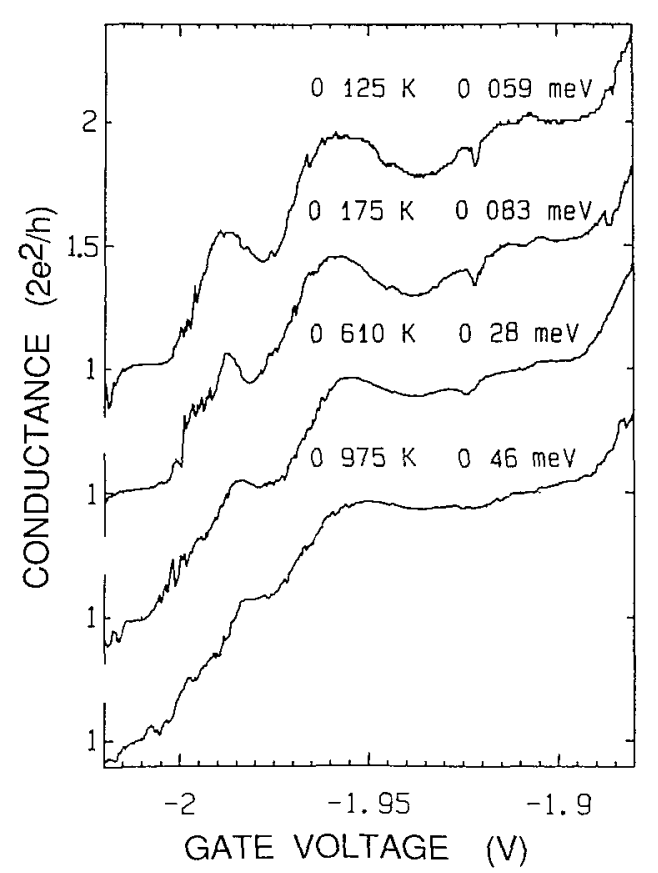

FIG. 4. Temperature averaging of the transmission resonances of the second subband. The values for the energyaveraging parameter $\Delta E$ are given. The curves have been offset for clarity. 


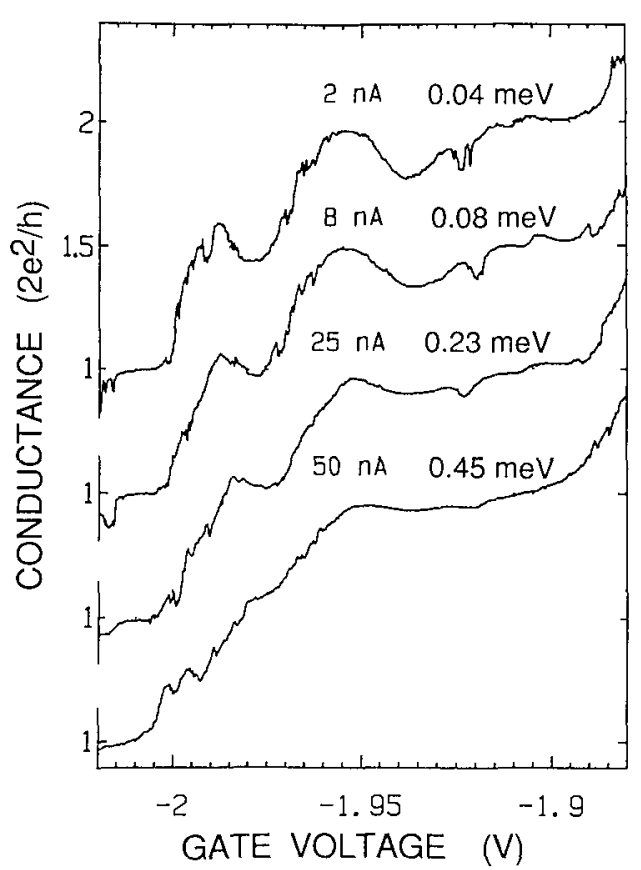

FIG. 5. Voltage averaging of the transmission resonances of the second subband. The values for the energy-averaging parameter $\Delta E$ are given. The curves have been offset for clarity.

subband spacing $E=\hbar \omega_{0} \approx 2.5 \mathrm{meV}$ (see Sec. III F). From the resonance condition $2 k_{y 1} L=3(2 \pi)$, we find $L \approx 140 \mathrm{~nm}$, which is a reasonable value, considering the width of the depletion regions around the gates, which is estimated to be about $200 \mathrm{~nm}$.

We emphasize that, although several devices showed structure in the transition region between the plateaus, clear resonances have been observed in only two devices. We will discuss this further in Sec. III E.

\section{Energy averaging of the conductance}

In the preceding sections it was shown that at low voltages across the device and low temperatures the conductance of a QPC can be described by the transmission probabilities $T_{n}\left(E_{F}\right)$ of the different subbands at the Fermi energy. At a finite temperature, or finite voltage across the device, the current will be carried by an energy interval of finite width. This leads to energy averaging of the point-contact conductance. ${ }^{31}$ The conductance at a finite voltage $V$ is given by

$$
G_{c}(V)=\frac{2 e}{h} \frac{1}{V} \sum_{n=1}^{N_{c}} \int_{E_{\Gamma}}^{E_{1}+e V} T_{n}(E) d E
$$

At a finite temperature $T$ the conductance is given by

$$
G_{c}(T)=\frac{2 e^{2}}{h} \sum_{n=1}^{N_{c}} \int_{0}^{\infty}\left(\frac{d f(E, T)}{d E}\right) T_{n}(E) d E,
$$

in which $f(E, T)=\left[1+\exp \left(E-E_{F}\right) / k T\right]^{-1}$ is the Fermi-Dirac distribution function. Equations (7) and (8) show that in both cases the physics is the same, and only the weighing factors are different. The temperature averaging has a Gaussian weighing factor, which has an effective width $\Delta E \approx 4 k_{B} T$. For voltage averaging, $\Delta E=e V$.

We have compared experimentally the effect of voltage and temperature averaging on the transmission resonances in the QPC conductance. Figure 4 shows the disappearance of the resonances when the temperature is increased, and Fig. 5 shows how they disappear when the ac current through the device is increased. The currents and temperatures have been selected such that each set of traces has approximately the same energy-averaging parameter $\Delta E$. (The effective $\Delta E$ due to the ac current with the rms value $I$ is estimated to be $\Delta E \approx 1.4 e I / G_{c}$.) The results show that the effects of elevated temperature and voltage are similar. The transport remains ballistic, at least up to temperatures of $1 \mathrm{~K}$ and voltages of $0.4 \mathrm{mV}$ across the device. Recent experiments show that ballistic and phase-coherent transport in a 2DEG can even occur up to energies in the meV range. ${ }^{16,32}$

Figures 4 and 5 show that an energy interval $\Delta E \approx 0.5$ $\mathrm{meV}$ is sufficient to wash out the transmission resonances. We now investigate how the quantized plateaus themselves are destroyed when the temperature is raised further. Figure 6 shows that temperature averaging becomes effective above $\approx 0.6 \mathrm{~K}$. At $4.2 \mathrm{~K}$ the plateaus have almost disappeared. The mechanism for the destruction of the plateaus is that at high temperatures electron states of the next subband become occupied, and not all electron states of the low-lying subbands are fully occupied anymore [Eq. (8)]. A comparison of the effective energy-averaging parameter at $4.2 \mathrm{~K}, \Delta E \approx 1.6 \mathrm{meV}$ with the subband spacing obtained in Sec. III F ( $\approx 2.5 \mathrm{meV}$ ), confirms that the mechanism for the destruction of the quantized plateaus is energy averaging. ${ }^{31}$ The $4.2-\mathrm{K}$ trace shows that the plateaus near pinchoff are less rounded than the other plateaus. ${ }^{33}$ This is in agreement

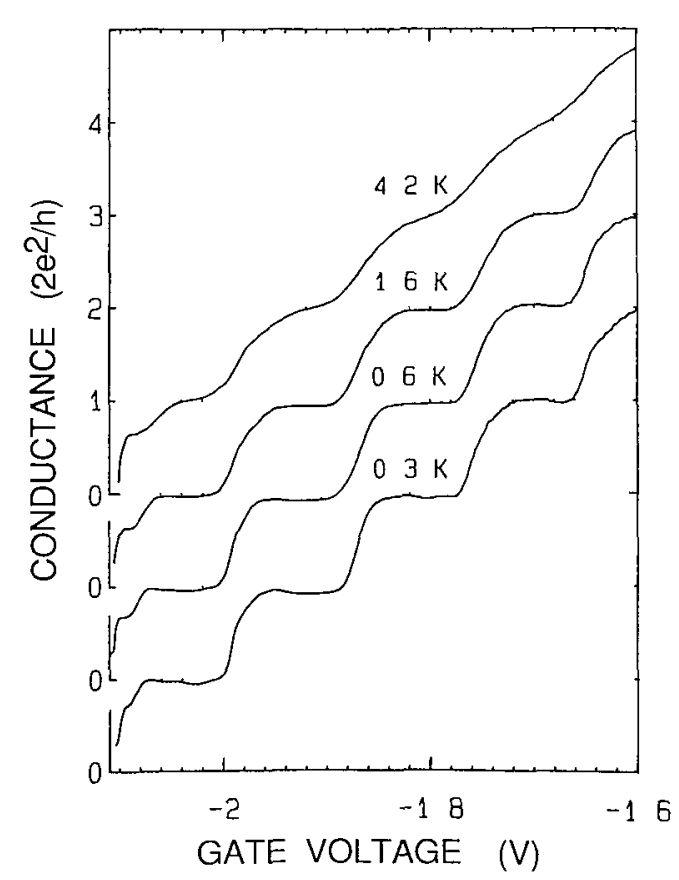

FIG. 6. Breakdown of the conductance quantization due to temperature averaging. The curves have been offset for clarity. 
with Sec III F, which shows that the subband spacing increases when the gate voltage is reduced Finally, we mention that the breakdown of the conductance quant1zation as a function of applied voltage has been studied by Kouwenhoven et al ${ }^{17}$ They showed that the conductance quantization breaks down at a voltage that is approximately equal to the subband spacing

\section{E. Comparison of the experimental results with model calculations}

After the discovery of the quantized conductance of point contacts, many calculations of the conductance of narrow constrictions have been performed ${ }^{34}{ }^{60}$ In this section we make a comparison between these model calculations and our experimental results We do not give an exhaustive discussion, but focus on the aspects that are relevant for the experimental results

An interesting question is whether an actual channel of finite length is required to observe quantization of the conductance, or whether a "hole-1n-a-screen" point contact is already sufficient Calculations ${ }^{36} 394445$ show that the conductance of a "hole-in-a-screen" point contact, calculated as a function of its width $W$, already shows a modulation with a period $2 e^{2} / h$ van der Marel and Haanappe ${ }^{36}$ obtained the suiprising result that the conductance at the points of inflection in the $G_{c}(W)$ curve is exactly equal to multiples of $2 e^{2} / h$ When the point contact is given a finite length, the structure rapidly develops into well-defined plateaus It was found that the length $L$ of the channel should exceed $03 \sqrt{2 W \lambda_{I}}$, to prevent the contribution of evanescent waves to the conductance, which destroy the quantized plateaus However, strong transmission resonances are observed when the channel is made longer such that it can accommodate several wavelengths

Several authors have calculated the conductance of a constriction with the typical wedge geometry of the lithographic gate (Fig 1) that defines the QPC's 3952 No well-defined plateaus were observed in this geometry This clearly shows that the actual electrostatic potential that defines the QPC's is substantially different from the geometry of the lithographic gate The potential is the 2DEG changes more smoothly than the lithographic gate, and this impioves the quality of the quantization

If the change in width and electrostatic potential at both ends of the channel is sufficiently smooth, adiabatic transpoit can occur In this case the electrons move with conservation of subband index, and no mode mixing takes place Adiabatıc transport through QPC's was studied in Refs 37 and 41 Glazman et al obtaned a condition for the radius of curvature of the boundaries of the constriction, iequired for ddiabatic transport However, it is difficult to compare this criterion with the experimental results, since the actual QPC's also contan a potential barrier (see Sec III F), which is not included in the calculations

Several authors have included scatterng in therr model calculations, which, as expected, destroys the quantization Recently Nixon et al and Laughton et al ${ }^{34}$ calculated the transport through QPC's, by modeling the confining potential as the sum of the potential due to the gates and the fluctuating potential due to the randomly distributed donor atoms They find that QPC's with different donor distributions show a different quality of the quantized plateaus, as well as different fine structure in between the plateaus For particular potentials they find resonances in the conductance, similar to those described in Sec IIIC Although a slight variation in the gate geometry for different devices cannot be ruled out, we think that the reason that resonance structure is observed is due to the fact that backscattering at both ends of the channel may be enhanced by the fluctuating potential

\section{F. Transition from zero-field quantization to quantization in high magnetıc fields}

In this section we study the effect of a perpendicular magnetic field on the conductance quantization It is shown that the application of a magnetic field preserves the quantization and a gradual transition is observed from the conductance quantization, due to the lateral confinement of the electrons, to the quantization in high magnetic fields We deliberately do not use the term quantum Hall effect, since this is restricted to fourterminal measurements, whereas we study a two-terminal conductance However, as we will show, the origin of the quantum Hall effect and the zero-field quantization is closely related

The presence of a perpendicular magnetic field does not change the one-dimensional nature of the transport in the QPC Because of the translational invariance of the Hamiltonian in the direction along the channel, the transport can still be described by electron waves traveling in a waveguide The dispersion of these waves now becomes

$$
E_{n}\left(k_{y}\right)=\left(n-\frac{1}{2}\right) \hbar \omega+\frac{\hbar^{2} k_{y}^{2}}{2 m}+e V_{0} \pm 1, g \mu_{B} B
$$

with

$$
m=m * \frac{\omega^{2}}{\omega_{0}^{2}}, \quad \omega=\sqrt{\omega_{0}^{2}+\omega_{c}^{2}}, \quad \text { and } \omega_{c}=\frac{e B}{m^{*}}
$$

The magnetic field creates hybrid magnetoelectric subbands, and changes the dispersion relation of the waves ${ }^{61}$ However, because of the one-dimensional nature of the transport, the essential relation between the $1 \mathrm{D}$ density of states $N_{n}(E)$ and the group velocity $v_{n}(E)$ still holds $N_{n}(E) v_{n}(E)=4 / h$ Ignoung spin splitting we obtain the result ${ }^{62}$

$G_{c}(B)=\frac{2 e^{2}}{h} N_{c}$ with $N_{c}=$ int $\left(\frac{E_{F}-e V_{0}}{\hbar \omega}+\frac{1}{2}\right)$

Equation (10) shows that there is a gradual transition between the quantization in zero field $\left(\omega_{c}=0\right)$ to the quant1zation in high field $\left(\omega_{c} \gg \omega_{0}\right) 126364$

Figure 7 presents experimental results on the transition from zero-field to high-field quantization, ${ }^{12}$ obtained at $06 \mathrm{~K}$ The top trace reproduces the $B=0$ result When a magnetic field is applied, the width of the plateaus is 


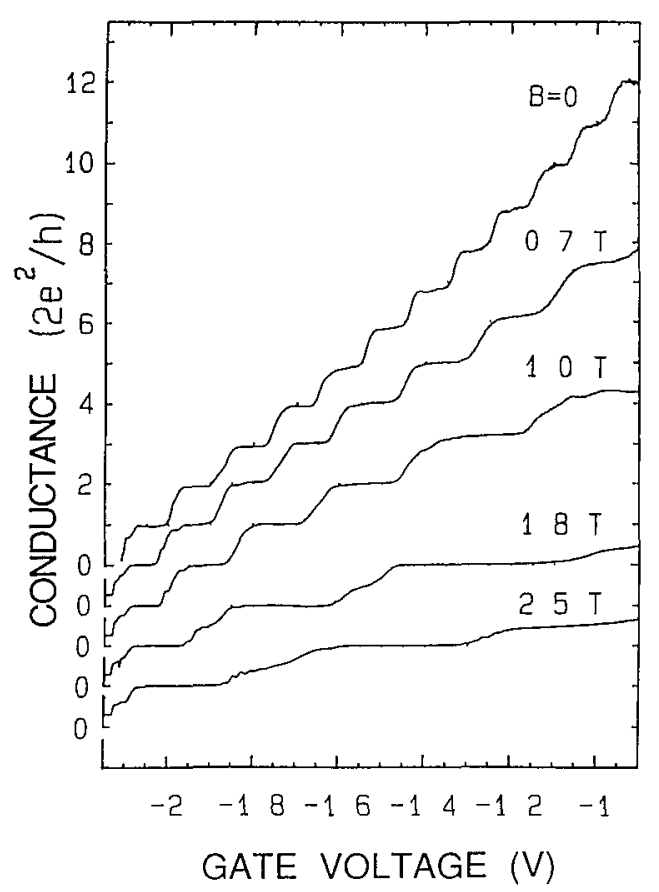

FIG. 7. Transition from quantization in zero field to quantization in high magnetıc fields, obtained at several fixed values of the magnetic field at $0.6 \mathrm{~K}$. The conductances were calculated from the measured resistances after subtraction of the resistances of the bulk contacts. The curves have been offset for clarity.

widened compared to the $B=0$ case. This reflects the increase of subband spacing with magnetic field [Eq. (9)]. It takes a larger variation of the gate voltage to populate (or depopulate) a new subband. The quantization is preserved, in agreement with Eq. (10). At high fields the spin degeneracy is lifted $\left(g \mu_{B} B\right.$ exceeds $\left.k_{B} T\right)$, and pla-

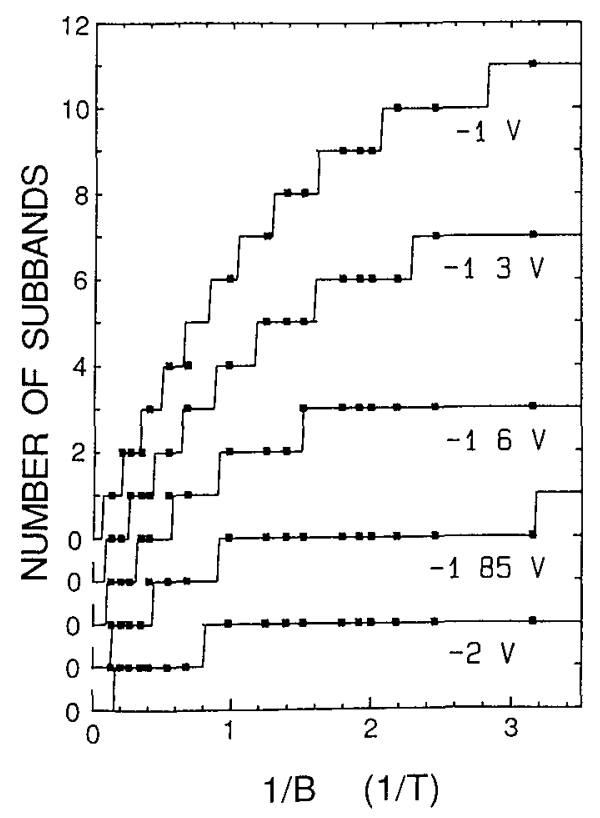

FIG. 8. Number of occupied subbands as a function of inverse magnetic field (square dots) obtained at several fixed values of the gate voltage. The solid curves correspond to fits with Eq. (9). The parameters are given in Table I. The curves have been offset for clarity.
TABLE I. Values for the subband spacing $\hbar \omega_{0}$ and potential barrier $e V_{0}$ at several values of the gate voltage $V_{g}$, obtained from a fit of Eq. (9) to the experimental data of Fig. 8.

\begin{tabular}{lcc}
\hline \hline$V_{g}(\mathrm{~V})$ & $\hbar \omega_{0}(\mathrm{meV})$ & $e V_{0}(\mathrm{meV})$ \\
\hline-1.0 & 1.0 & 0 \\
-1.3 & 1.1 & 2.0 \\
-1.6 & 1.5 & 3.5 \\
-1.85 & 1.8 & 5.5 \\
-2.0 & 3.0 & 6.5 \\
\hline
\end{tabular}

teaus at uneven multiples of $e^{2} / h$ become visible.

Equation (10) predicts that at high magnetic fields $\left(\omega_{c} \gg \omega_{0}\right), N_{c}$ is determined exclusively by the combination of the potential barrier $V_{0}$ and $\omega_{c}$, and is proportional to $1 / B$. At low fields, however, the number of subbands is limited by the lateral confinement, and determined by $\omega_{0}$. We have determined the number of occupied subbands $N_{c}$ as a function of magnetic field at several fixed values of the gate voltage from Fig. 7. The result is shown in Fig. 8 (square dots). From the fit of Eq. (10) to these data we have obtained the values of $V_{0}$ and $\omega_{0}$ at these values of the gate voltage. They are given in Table I (a similar analysis for an infinite square-well potential is given in Ref. 12). The results show that a reduction of the gate voltage increases both the confinement (measured by $\omega_{0}$ ) and the potential barrier $V_{0}$ in the QPC. The results show that the maximum subband spacing, which is achieved in our QPC's, is about $3 \mathrm{meV}$. Similar results have also been obtaned by Wharam et al. for a split-gate wire. ${ }^{65}$

A characteristic feature of QPC's in a magnetic field is that the quality of the quantization is improved when a magnetic field is applied. This is most clearly observed when the zero-field quantization is poor. In this case the

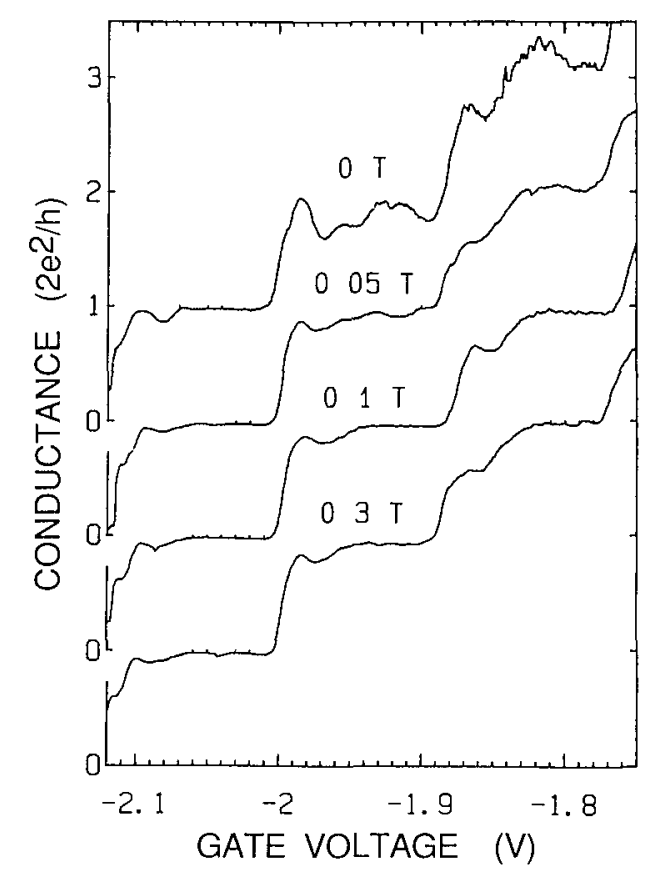

FIG. 9. Improvement of the conductance quantization by the application of a magnetic field, measured at $40 \mathrm{mK}$. 
quality of the quantization has deteriorated due to several thermal cycles Figure 9 shows how a relatively small magnetic field already improves the quantization The mechanısm is probably that the backscattering near or in the QPC is reduced in the presence of a magnetic field Because the quantization is already improved at a very low field (the cyclotron radius at $01 \mathrm{~T}$ is about $1 \mu \mathrm{m}$ ), it is possible that part of the backscattering occurs near the QPC (possibly by impurities), and not in the QPC itself

As discussed by Buttiker, ${ }^{6}$ a sufficiently large magnetic field can completely prevent the backscatteing induced by impurities or irregularities in the confining potential This absence of backscattering in high magnetic fields is probably the main reason for the extreme accuracy of the quantum Hall effect, compared to the limited accuracy of the conductance quantization in zero field

\section{G. Concluding remarks}

The conductance of quantum point contacts was found to display quantized plateaus at multiples of the conductance quantum $2 e^{2} / h$ This quantization can be explained by the formation of one-dimensional subbands in the point contacts, each occupied subband contributing $2 e^{2} / h$ to the conductance Both experiments and model calculations show that the accuracy of the quantization is sensitive to the detailed shape of the confining potential and the possible presence of impurities Nevertheless, we estimate that it may be possible to obtain accuracies exceeding $01 \%$ in properly designed geometries However, the fact that the quantization can probably be destroyed by a single impurity, located at an unfavorable position, will exclude the possible use of QPC's as a resistance standard

The experiments show that the transport thiough the QPC's remains ballistic up to at least $42 \mathrm{~K}$ This means that inelastic processes are not yet impoitant at $42 \mathrm{~K}$ The conductance quantization bi eaks down due to energy avelaging It is shown that the application of a magnetic field leads to a gradual transition to magnetic quantization The major difference between the quantization in the absence of a field and the quantum Hall effect is the nature of the scattering In the absence of a magnetic field, the backscattering from impurities or irregularities in the confining potential will destroy the quantization As discussed in the following section, backscattering is suppressed by a sufficiently high magnetic field

\section{QUANTUM TRANSPORT IN HIGH MAGNETIC FIELDS}

\section{A. A model for quantum transport in high magnetıc fields}

In this section we give a brief description of transport in high magnetic fields in a $2 \mathrm{DEG}$ free of imperfections We assume that the electrons are laterally confined in the 2DEG by the electrostatic potential given in Fig 10 The electrostatic potential $V(x)$ has a flat part in the middle, and rises at the edges of the $2 \mathrm{DEG}$ The width $W$ of these depletion regions at the edges is usually of the order of 100-500 $\mathrm{nm}$ in actual devices The following disper-
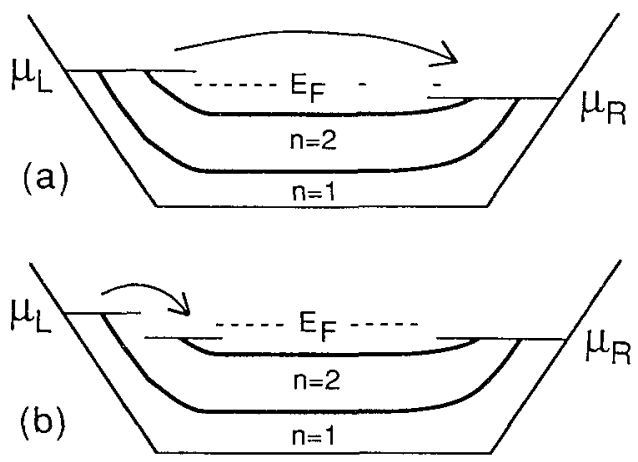

FIG 10 Cross section of a 2DEG, showing the occupied electron states of two Landau levels, in the presence of a current flow (a) shows the regulal situation The arrow indicates intra-Landau-level scattering (b) shows the occupred electron states when current is injected selectively with a QPC The arrow illustrates inter-Landau-level scatteıng between adjacent edge channels

sion relation is obtained for the electron states in the $2 \mathrm{DEG}^{5}$

$$
\begin{array}{r}
E_{n}\left(k_{y}\right)=e V(x)+\left(n-\frac{1}{2}\right) \hbar \omega_{\iota}+\frac{1}{2} m^{*} v_{D}^{2} \pm \frac{1}{2} g \mu_{B} B \\
v_{D}=\frac{E}{B}
\end{array}
$$

The energy of an electron consists of four terms the electrostatic energy $e V(x)$ at the center coordinate $x=l_{b}^{2} k_{y}$ of the electron wave function $\left(l_{b}=\sqrt{\hbar / e B}\right)$, the quantized cyclotron energy ( $n$ is the Landau-level index), the kinetic energy associated with the drifting motion of the electrons in crossed $E$ and $B$ fields, and the Zeeman spin-splitting term Evaluation of the third term in (11) with a typical value $E=E_{F} /(\mathrm{eW}) \approx 10^{4}-10^{5} \mathrm{~V} / \mathrm{m}$ for the electric field at the boundary of the $2 \mathrm{DEG}$ shows that this term can usually be neglected in high magnetic fields $(B>1 \mathrm{~T})$

The relevant electrons for transport are those at the Fermi energy $E_{\Gamma}$ We now obtain a very simple picture for electron tiansport when we note that electrons $w 1^{\text {th }}$ different Landau-level indices $n$ flow along different equipotential lines $V(x)$, which are given by the condition

$$
e V(x)=E_{F}-\left(n-\frac{1}{2}\right) \hbar \omega_{c} \pm \frac{1}{2} g \mu_{B} B
$$

Because this condition is usually satisfied at the edges of the 2DEG one speaks about transport in edge channels These edge channels are located at the intersections of the Landau levels and the Fermi energy Figure 10(a) shows the occupred electron states of two Landau levels when a net current $I$ flows in the 2DEG This current is a result of the difference in occupation of the right- and left-hand edge channels, which caily current in opposite directions It can be shown ${ }^{6} 8$ that the net current $I$ is independent of the details of the dispersion of the Landau levels and is given by

$$
I=N_{L} \frac{e}{h}\left(\mu_{L}-\mu_{R}\right)
$$

The current carried by each Landau level is simply given 
by $e / h$ multiplied by the electrochemical potential difference $\mu_{L}-\mu_{R}$ between right and left edge channels Voltage probes attached to either side of the 2DEG will measure this electrochemical potential difference, and the Hall resistance is

$$
R_{H}=\frac{V_{H}}{I}=\frac{\left(\mu_{L}-\mu_{R}\right)}{e I}=\frac{e^{2}}{h} \frac{1}{N_{L}}
$$

This is the elementary explanation for the quantum Hall effect ${ }^{4}{ }^{8}$ A necessary condition for the observation of the QHE is that the right-hand contact exclusively measures the electrochemical potential of the right-hand edge channels and vice versa A second condition is that (back)scattering between the two sets of edge channels on either side of the $2 \mathrm{DEG}$ is absent

A major deficiency of the above description is that it does not take into account screening A description of the transpoit in terms of edge channels is only possible by assuming that the Fermi level $E_{\Gamma}$ in the interior of the $2 \mathrm{DEG}$ can be positioned in between the flat parts of two consecutive Landau levels In a $2 \mathrm{DEG}$ without potential fluctuations this is not possible, because the electron density is fixed, and the Fermi level will be pinned to the upper Landau level Calculations of self-consistent screening, which take into account the finite width of the 2DEG, support this picture 6667 They show that the large degeneracy of the Landau levels can result in perfect screening, and the Fermi level may be pinned to the upper Landau level in a considerable region of the 2DEG Because all electron states of the low-lying Landau levels remain occupied in the interior of the $2 \mathrm{DEG}$, the edge-channel description will remain valid for these Landau levels

Our experiments show that we can use the edgechannel description for all Landau levels, including the upper Landau level We think that this is due to the presence of potential fluctuations in the 2DEG These fluctuations will localize states in the bulk of the $2 \mathrm{DEG}^{5}$ As illustrated in Ref 6, this localization may be env1saged as edge channels that close upon themselves, and therefore do not affect the transport Although the edge-channel picture provides the basis for the understanding of the QHE, it is clear that a further investigation of the scattering processes in the bulk is required for a complete understanding of the QHE

\section{B. Hıgh-magnetıc-field transport in quantum point contacts}

The transport properties of QPC's in zero and nonzero magnetic field have been discussed in Sec III In this section we focus on the high-field regime, in which the electron transport can be described in terms of edge channels ${ }^{68}$ We first note that the electrostatic potential landscape at the QPC's has a saddle shape Besides the lateral confinement of the electrons, the potential in the QPC's is also raised relative to the bulk $2 \mathrm{DEG}$ This potential bartier $V_{0}$ is a function of the applied gate voltage (see Sec III F) In high magnetic fields (when $\omega_{c} \gg \omega_{0}$ ), the ti ansport is exclusively deter mined by $V_{0}$ and $\omega_{c}$, and independent of $\omega_{0}$ The number of occupied Landau levels in the QPC is reduced relative to the bulk and is given (a)
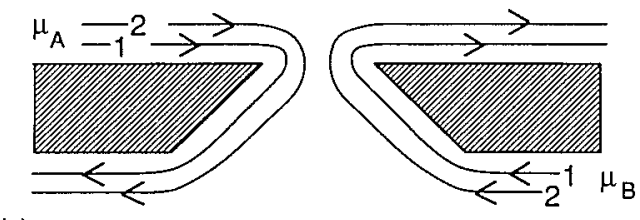

(b)

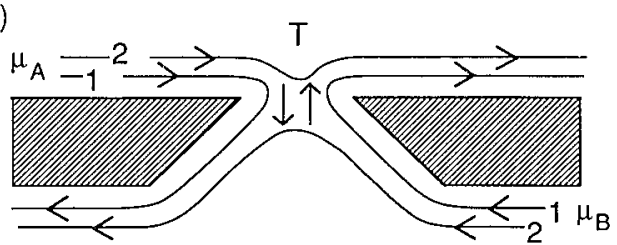

(c)

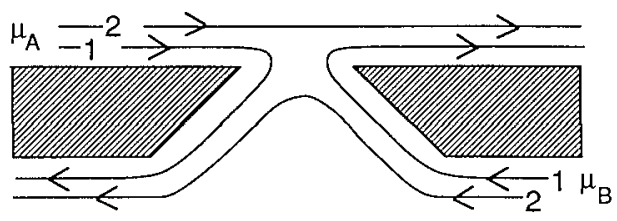

FIG 11 High magnetıc-field transport in a QPC for three different values of the potential barrier $V_{0}$, illustrated for the case of two occupred Landau levels (see text)

by $N=\operatorname{lnt}\left[\left(E_{F}-e V_{0}\right) /\left(\hbar \omega_{c}\right)+\frac{1}{2}\right]$

Figure 11 illustrates the current flow in edge channels through the QPC for three different values of the potential barrier $V_{0}^{9}$ In Fig $11(\mathrm{a})$ no potential barrier is present, and all edge channels are transmitted The QPC does not influence the electron transport This is approx1mately the case when the QPC is formed at $-06 \mathrm{~V}$ In Fig 11(b) the gate voltage is reduced, and a potential barrier is created In this particular example, a fraction $T$ of the electrons in the second edge channel is transmitted through the QPC and a fraction $R=1-T$ is reflected Note that the electrons in the edge channel with the highest Landau-level index are the first to be reflected, since this edge channel follows the lowest equipotential line In Fig 11(c) the potential barrier is such that this edge channel is completely reflected, whereas the other is still completely transmitted We now write the twoterminal resistance $G_{c}$ of the QPC as

$$
G_{c}=\frac{e I}{\mu_{A}-\mu_{B}}=\frac{2 e^{2}}{h}(N+T)
$$

In this expression, $N$ denotes the number of (spindegenerate) edge channels that are fully transmitted through the QPC, and $T$ denotes the transmission of the partially transmitted edge channel We assume that at the QPC only one edge channel can be partially transmitted, and all otheis are either completely reflected or completely transmitted Also we assume that no scattering between edge channels occurs in or near the QPC The observation of an anomalous integer quantum Hall effect (Sec IV C) shows that these assumptions are justified for $B>15 \mathrm{~T}$ in our device geometry

By considering the edge channels that flow away from the QPC, it can be seen that they are occupied up to different electrochemical potentials $\mu_{A}$ or $\mu_{B}$, depending on whether they have been transmitted or reflected at the 
QPC [see Fig. 11(c)]. This means that a QPC, when used as a current probe, can selectively inject current into only those edge channels that are transmitted by the QPC. Similarly, when used as a voltage probe, a QPC will exclusively measure the electrochemical potentials of those edge channels that are transmitted through the QPC.

\section{Anomalous integer quantum hall effect}

In this section we investigate the (quantization of the) Hall conductance, when it is measured with QPC's that couple selectively to specific edge channels. In the regular QHE, when the Hall conductance is measured with ideal bulk contacts (which couple ideally to all available $N_{L}$ edge channels), the quantization of $G_{H}$ is determined by the number of bulk Landau levels $N_{L}$. The formation of a quantized plateau in $G_{H}$ is accompanied by a vanishing of the longitudinal resistance $R_{L}$. It is shown in this section that the selective coupling of the QPC's, combined with the absence of scattering between edge channels, leads to an anomalous quantization of the Hall conductance, in which $G_{H}$ is not determined by the number of bulk Landau levels $N_{L}$, but by the number of Landau levels in the QPC's instead. ${ }^{9}$ At the same time, the longitudinal resistance shows quantized plateaus (see Sec. IV D). We emphasize that the anomalous quantization of the Hall and longitudinal resistances, as well as the adiabatic transport in series QPC's (Sec. IV D) have the same origin: the selective population and detection of edge channels, combined with the absence of scattering between edge channels in the region between the QPC's.

In an identical device, van Houten et al..$^{13-15}$ studied coherent electron focusing at low fields. Electronfocusing peaks were observed in both Hall and longitudinal resistances as a result of the ballistic transport in skipping orbits between the QPC's. At low fields many edge channels are occupied, and the focusing peaks can be explained with a classical calculation. The fine structure in the focusing spectrum was explained by the quantum interference between many coherently excited edge channels. ${ }^{14,15}$ In this paper we are interested in the highfield regime, where only a few edge channels are occupied.

We calculate $G_{H}$, which is defined as the ratio of the current $I$ and the voltage difference between contacts 1 and 6 , when 5 and 4 are used as current probes [see Figs. 1 and 12(a)]. The two QPC's serve as adjacent current and voltage probes. We first perform the calculation for a forward-directed magnetic field. We assume that all bulk contacts are ideal. This means that these contacts absorb the total current that flows along the 2DEG boundary, and that all $N_{L}$ edge channels that leave a bulk contact are equally occupied and have the same electrochemical potential. ${ }^{6}$ An ideal contact, therefore, has a two-terminal conductance $G=\left(2 e^{2} / h\right) N_{L}$. In the calculation we set $\mu_{1}=0$ for convenience. By employing the general Büttiker formula for four-terminal measurements, ${ }^{69}$ an expression for $G_{H}$ can be given in terms of transmission probabilities between the bulk contacts. However, we prefer to give a step-by-step derivation of the result, which brings out the physics involved more (a)

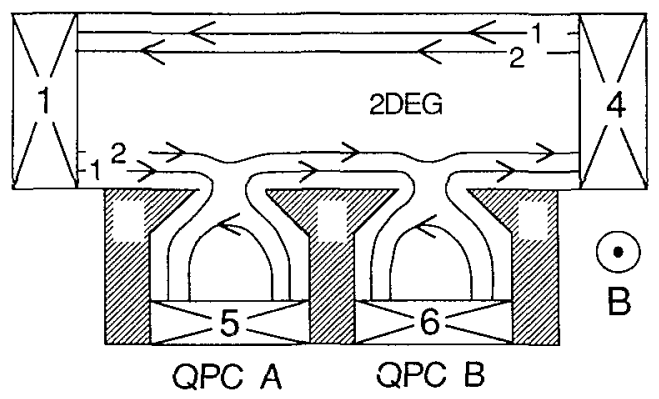

(b)

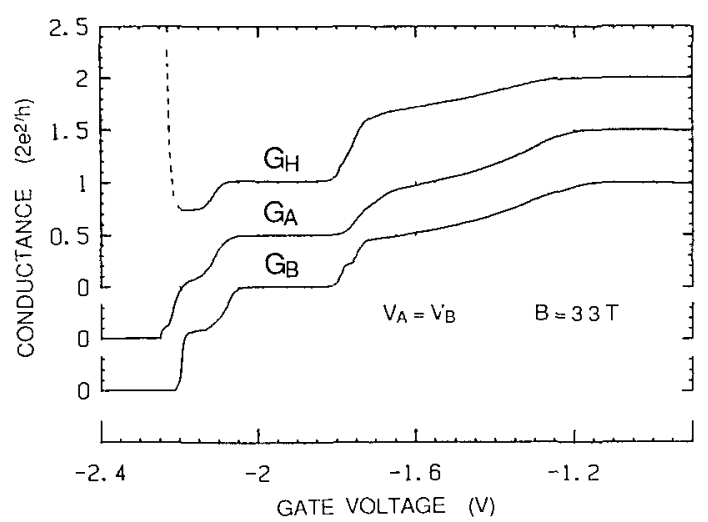

FIG. 12. (a) Electron flow in edge channels, resulting in an anomalous quantization of the Hall conductance $G_{H}=2 e^{2} / h$. (b) Comparison between the two-terminal conductances $G_{A}$ and $G_{B}$ of the point contacts with the Hall conductance $G_{I I}$. The Hall conductance shows an anomalous plateau at $2 e^{2} / h$, in agreement with Eqs. (17)-(20). The rapid rise in $G_{H}$ below $-2.2 \mathrm{~V}$ is an artifact due to the complete pinchoff of the QPC's. The curves have been offset for clarity.

clearly.

The two-terminal conductance of the current QPC $A$ can be written

$$
G_{A}=\frac{e I}{\mu_{5}-\mu_{1}}=\frac{2 e^{2}}{h}\left(N_{A}+T_{A}\right),
$$

in which $N_{A}$ denotes the number of fully transmitted (spin-degenerate) edge channels, and $T_{A}$ denotes the transmission of the partially transmitted edge channel through QPC $A$. Whenever $N_{A}<N_{L}$, the injected current is disturbed unequally over the available $N_{L}$ bulk edge channels [Fig. 12(a) illustrates the electron flow for the $N_{L}=2$ case, and $\left.N_{A}, N_{B}=1\right]$. The lowest $N_{A}$ channels are fully occupied up to $\mu_{5}$, and carry a current $(2 e / h) N_{A} \mu_{5}$. Channel $N_{A}+1$ is only partially occupied, and carries a current $(2 e / h) \mu_{5} T_{A}$. Channels $N_{A}+2$ up to $N_{L}$ are not populated at all, and carry no current. The injected current flows towards the voltage QPC $B$. At this point we assume that no scattering between edge channels takes place in the region between the QPC's.

In order to calculate $\mu_{6}$ and $G_{H}$, we have to consider three situations. When $N_{B}>N_{A}\left(N_{B}\right.$ is the number of fully transmitted edge channels by QPC $B$ ), the total injected current $I$ will enter the voltage QPC $B$. Because 
QPC $B$ is a voltage contact, an electrochemical potential $\mu_{6}$ will build up to compensate this in-going current with an equal out-going current. This electrochemical potential is determined by the two-terminal conductance $G_{B}$ and is given by $\mu_{6}=e I / G_{B}$. This yields the Hall conductance:

$$
G_{H}=G_{B}=\frac{2 e^{2}}{h}\left(N_{B}+T_{B}\right) \text { for } N_{B}>N_{A} .
$$

In the $N_{A}>N_{B}$ case, all channels entering the voltage QPC are fully occupied up to $\mu_{5}$. This means that $\mu_{6}$ becomes equal to $\mu_{5}$ and

$$
G_{H}=G_{A}=\frac{2 e^{2}}{h}\left(N_{A}+T_{A}\right) \text { for } N_{A}>N_{B}
$$

If $N_{A}=N_{B}=N$, the current entering the voltage probe as a result of the fully populated channels is given by $(2 e / h) N \mu_{5}$. Channel $N+1$ carries a current $(2 e / h) T_{A} \mu_{5}$, of which an amount $(2 e / h) T_{A} T_{B} \mu_{5}$ enters the voltage probe. Compensation of the total in-going current by an equal out-going current gives the result

$$
G_{H}=\frac{2 e^{2}}{h} \frac{\left(N+T_{A}\right)\left(N+T_{B}\right)}{\left(N+T_{A} T_{B}\right)} \text { for } N_{A}=N_{B}=N .
$$

Equations (17)-(19) predict that $G_{H}$ is quantized whenever the QPC with the largest conductance is quantized. The quantized values for $G_{H}$ are given by

$$
G_{H}=\frac{2 e^{2}}{h} \max \left(N_{A}, N_{B}\right) \text {. }
$$

The fact that the number of bulk Landau levels $N_{L}$ does not appear in the equations for $G_{H}$ can be understood by the fact that a bulk edge channel that is neither populated by QPC $A$ nor detected by QPC $B$ is irrelevant for the electron transport.

The anomalous QHE will be destroyed by scattering between populated and nonpopulated edge channels in the region between the QPC's. The regular QHE does not require the absence of scattering between adjacent edge channels. In this case, all edge channels located at a given boundary of the $2 \mathrm{DEG}$ are in equilibrium, and all have the same electrochemical potential. This means that the scattering rate from one edge channel to another is perfectly compensated for by an equal scattering rate in the opposite direction.

In a reverse magnetic field the electrons that are injected by QPC $A$ move away from QPC $B$ and flow towards bulk contact 1 . We have assumed that this contact is ideal, which means that it can be represented as a contact with a two-terminal conductance $G=\left(2 e^{2} / h\right) N_{L}$. The Hall conductance now has the regular value $G_{H}=\left(2 e^{2} / h\right) N_{L}$ (it is determined by the probe with the largest conductance). We therefore see that in reverse field the properties of bulk contact 1 are important for the establishment of the regular quantum Hall effect. Büttiker ${ }^{6}$ has suggested that in the case of nonideal contacts (which do not couple ideally to all $N_{L}$ edge channels) a regular quantum Hall effect may still occur, pro- vided that the edge channels are equilibrated by inelastic scattering in between the contacts. However, it is shown in Sec. IV F that under certain circumstances scattering between adjacent edge channels can be weak even on macroscopic length scales $(>200 \mu \mathrm{m})$, which implies that the properties of bulk contacts may be important for the establishment of the QHE. ${ }^{70}$

We have measured the Hall conductance $G_{H}$, as well as $G_{A}$ and $G_{B}$, as a function of magnetic field at $1.3 \mathrm{~K}$ for several fixed values of the gate voltage $\left(V_{A}=V_{B}\right)$. In reverse magnetic fields the regular $\mathrm{QHE}$ is observed. The number of observed plateaus, as well as their positions, are not affected by the gate voltage. Figure 12(b) presents results obtained in forward magnetic field. A comparison is made between the two-terminal conductances $G_{A}$ and $G_{B}$, measured between contact pairs 1-5 and 1-6, respectively, and the Hall conductance $G_{H}$. Two bulk Landau levels are occupied at $3.3 \mathrm{~T}$. The measured Hall conductance closely follows the probe conductances, and exhibits an anomalous plateau at $2 e^{2} / h$. The rapid rise of $G_{H}$ below $-2.2 \mathrm{~V}$ is an artifact due to the complete pinchoff of the QPC's. These results are consistent with Eqs. (17)-(20), and provide the experimental proof of the selective population and detection of edge channels by the QPC's. In addition, the accurately quantized anomalous plateau implies that the scattering between edge channels is extremely weak, and that adiabatic transport takes place between the QPC's.

We have made a comparison between the probe conductances and the Hall conductance for a range of fixed magnetic fields. The results are presented in Fig. 13. The

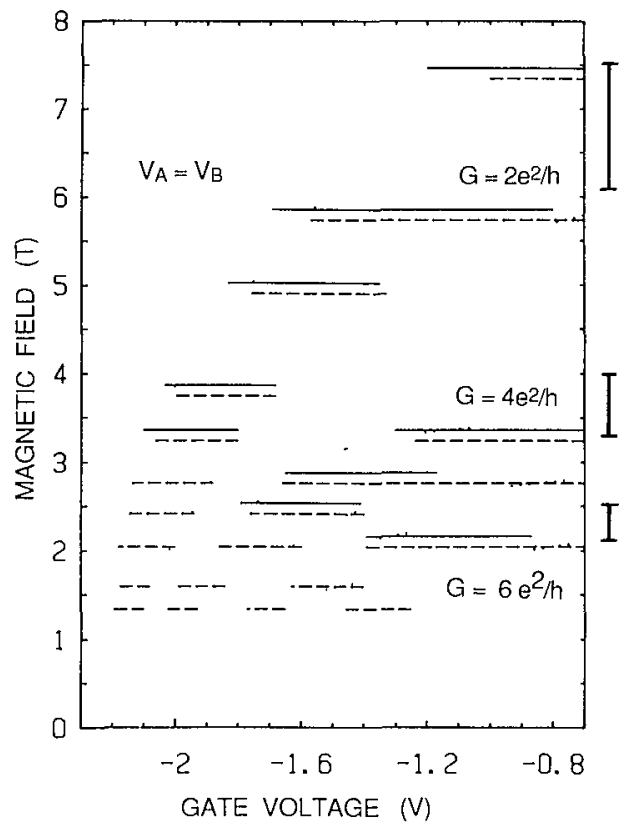

FIG. 13. Comparison between the quantization of $G_{A}$ and $G_{B}$ and the quantization of $G_{H}$ for several fixed values of the magnetic field. The dashed lines indicate the gate-voltage intervals in which both $G_{A}$ and $G_{B}$ are quantized. Solid lines indicate the gate-voltage intervals in which $G_{H}$ is quantized (see text). The dashed and solid lines have been offset for clarity. The magnetic-field intervals in which the regular quantum Hall plateaus occur are indicated at the right-hand side of the figure. 
dashed lines indicate the gate-voltage intervals in which $G_{A}$ and $G_{B}$ deviate less than $0.05\left(2 e^{2} / h\right)$ from the corresponding quantized values. The accompanying solid lines indicate the intervals in which $G_{H}$ shows quantized plateaus, obtained with the same criterion. These results confirm that the quantization of $G_{H}$ is determined by the quantization of the probe conductances. (For comparison we have indicated the magnetic-field intervals in which the regular quantum Hall plateaus occur at the right-hand side of the figure. This was measured with regular bulk contacts, by applying no voltage to the gates.)

At low fields $(B<2.0 \mathrm{~T}), G_{H}$ measured in forward fields fails to show quantized plateaus, whereas the probe conductances are already quantized for $B>1.4 \mathrm{~T}$ (the QPC's in this particular sample show poor quantization in the absence of a field, and therefore require a magnetic field to improve the quantization). We attribute this to the onset of inter-edge-channel scattering at low fields. This probably occurs at the exit of the QPC $A$ and the entrance of QPC $B$, where the confining electrostatic potential changes rapidly. It can be seen in Fig. 13 that at low gate voltages a higher magnetic field is required to obtain an anomalously quantized plateau. This may be due to the fact that the presence of a higher potential barrier $e V_{0}$ at low gate voltages (see Sec. III G) increases the scattering rate between edge channels, and therefore a higher field is required to obtain adiabatic transport. Note that the quantization of the two-terminal conductance of a QPC is not affected by scattering between adjacent edge channels that flow in the same direction.

At even lower fields $(B<1.0 \mathrm{~T})$, electron-focusing peaks are observed. At low temperatures large quantum interference effects have been observed in $G_{H} \cdot{ }^{13,14}$ This means that no adiabatic transport occurs in low fields. QPC $A$ excites several edge channels coherently, which subsequently gives rise to interference, since QPC $B$ also couples coherently to several edge channels.

The role of an individual QPC has been investigated by fixing both magnetic field and gate voltage $V_{B}$. In this way, $N_{L}$ and $G_{B}$ are kept constant. Figure 14(a) gives a comparison between $G_{H}$ and $G_{A}$, both measured as a function of $V_{A}$. $G_{B}$ has been fixed at $4 e^{2} / h$. The number of occupied Landau levels $N_{L}=3$. Whereas $G_{A}$ drops from the $4 e^{2} / h$ to the $2 e^{2} / h$ plateau, $G_{H}$ remains quantized at $4 e^{2} / h$. This illustrates that $G_{H}$ remains quantized whenever the QPC with largest conductance ( $G_{B}$ in this case) remains quantized [Eq. (17) with $N_{B}=2$ and $\left.T_{B}=0\right]$. In Fig. 14(b), $G_{B}$ has been fixed at $2 e^{2} / h$. The number of occupied Landau levels $N_{L}=2$. For $G_{A}>G_{B}$, the Hall conductance reproduces the features present in $G_{A}$. For $G_{A}<G_{B}$ the Hall conductance remains fixed at $2 e^{2} / h$, until QPC $A$ is fully pinched off. These observations correspond with Eq. (19) $\left(N_{A}, N_{B}=1, T_{A} \neq 0\right.$, $\left.T_{B}=0\right)$ and Eq. (17) $\left(N_{B}=1, T_{B}=0\right)$, respectively. These experiments confirm that the anomalous integer QHE is a result of the nonlocal transport. The Hall conductance changes, although the 2DEG in between the QPC's is not affected ( $V_{B}$ as well as $B$ are constant).

Equations (17)-(20) have been derived assuming spin
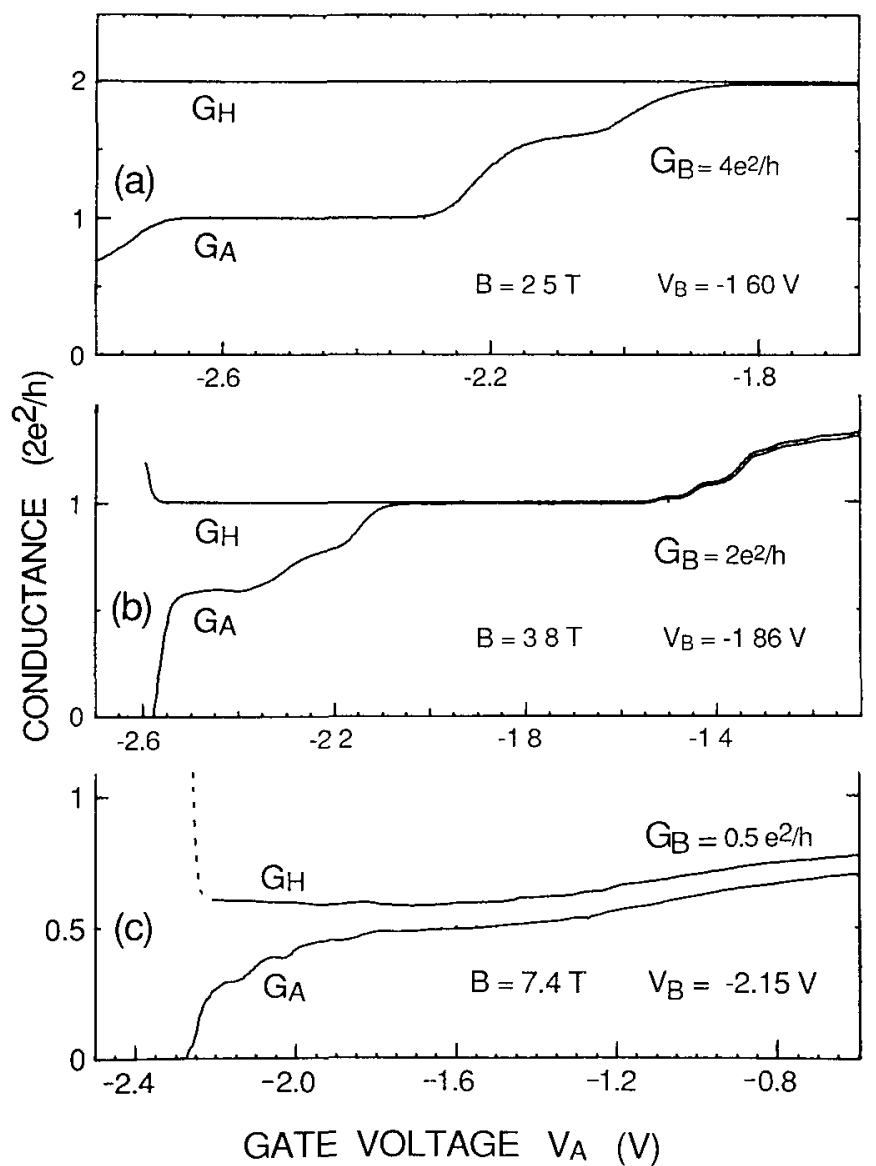

FIG. 14. (a) and (b) Comparison between the Hall conductance $G_{H}$ and the conductance of the current probe $G_{A}$, demonstratıng the validity of Eqs. (17)-(20). (c) Comparison between $G_{A}$ and $G_{I I}$, illustrating the saturation of $G_{I I}$ near $e^{2} / h$.

degeneracy. At high fields, when the spin splitting is resolved (due to the Zeeman splitting $g \mu_{B} B$ exceeding $k_{B} T$ ), these equations remain valid provided that the conductance quantum is replaced by $e^{2} / h$, and $N$ and $T$ apply to single-spin channels. Equation (19) shows that there is an interesting exception to the rule that the quantization of $G_{H}$ is determined by the largest QPC conductance. It predicts that when the current and voltage QPC couple to one (single-spin) channel only $\left(N_{A}, N_{B}=0\right), G_{H}$ is always quantized at $e^{2} / h$. This means that the anomalous Hall conductance, measured in forward fields, cannot drop below $e^{2} / h$. A similar result has been obtained by Sivan, Hartzstein, and Imry. ${ }^{71}$

We have investigated this experimentally by applying a fixed gate voltage $V_{B}$ such that the resistance of QPC $B$ is high $(\approx 50 \mathrm{k} \Omega)$, which implies $T_{B} \approx 0.5$. In Fig. 14(c) a comparison is given between the Hall conductance $G_{I I}$ and $G_{A}$, both measured as a function of $V_{A}$. When QPC $A$ is slowly pinched off, the Hall conductance saturates at $\approx 1.2 e^{2} / h$ and remains at that value until the complete pinchoff of QPC $A$. The fact that $G_{H}$ does not fully reach the value $e^{2} / h$ is probably due to the fact that the spin splitting is not yet complete at $1.3 \mathrm{~K}$ (QPC $B$ shows a "quantized" plateau at $\left.G_{B} \approx 1.2 e^{2} / h\right)$. In the same experiment, performed at $100 \mathrm{mK}$, the Hall conductance 
indeed saturates at the $e^{2} / h$ plateau.

The above results support the picture that transport in the (integer) quantum Hall regime takes place through edge channels, which each have a conductance $e^{2} / h$. Recently the fractional quantum Hall regime was studied with similar devices. ${ }^{72}$ While the filling factor in the bulk 2DEG was kept fixed at $v=1$, a Hall conductance $G_{H}=\frac{2}{3} e^{2} / h$ was observed, when the filling factor in the adjacent voltage and current probes was reduced. The experiment implies that a description in terms of (fractional) edge channels is valid in the fractional quantum Hall regime as well. ${ }^{73}$

Glazman and Jonson ${ }^{74}$ have obtained a criterion for adiabatic transport in high magnetic fields. They modeled the QPC's by a hard-wall potential boundary. Scattering between edge channels is induced when the boundary has a finite radius of curvature $R$. With an estimate of $R \approx 0.2 \mu \mathrm{m}$ for the radius of curvature of the 2DEG boundary near the entrance and exit of the QPC's, they obtained a threshold field of $B \approx 1.0-1.5 \mathrm{~T}$, required to suppress the inter-edge-channel scattering. Although this value is quite near the experimentally observed threshold fields, we think that the presence of a potential barrier in the QPC's will also affect the adiabatic transport, and should also be included in the calculations.

Recently it was suggested ${ }^{75,76}$ that the reduction of the spatial overlap of the wave functions of adjacent edge channels can be an important factor in the suppression of the inter-edge-channel scattering. In high magnetic fields, the wave functions decay as $\exp \left[-\left(\Delta y / l_{b}\right)^{2}\right]$, with $\Delta y$ the distance from the center of the wave function and $l_{b}$ the magnetic length. The overlap of the wave functions therefore becomes exponentially small when the separation of the centers of the wave functions becomes larger than the magnetic length. From an estimated width $W \approx 150 \mathrm{~nm}$ for the depletion regions we obtain a typical value for the electric field $E=E_{F} /(e W) \approx 8 \times 10^{4}$ $\mathrm{V} / \mathrm{m}$ at the boundaries of the 2DEG. At $B \approx 2.0 \mathrm{~T}$ (the typical field required for adiabatic transport) the separation of the wave functions of adjacent edge channels is estimated to be $\hbar \omega_{c} /(e E) \approx 35 \mathrm{~nm}$. At this magnetic field, $l_{B} \approx 17 \mathrm{~nm}$. This shows that the overlap of the wave functions is indeed reduced when adiabatic transport occurs.

In this section we have used QPC's to simulate nonideal contacts, which do not couple equally to all $N_{L}$ edge channels. We have shown that, because of the lack of equilibration between the edge channels, these nonideal contacts give rise to deviations from the regular QHE, and can even result in an anomalous QHE. Komiyama et $a l .{ }^{70}$ have studied the deviations from the regular QHE that occur in samples with nonideal bulk contacts. In their case, a nonequilibrium population of edge channels is created by the backscattering at a cross gate. They find that at $B=3.8 \mathrm{~T}$ the nonequilibrium population of the edge channels created by the backscattering at the cross gate can considerably affect the Hall voltage that is measured about $50 \mu \mathrm{m}$ away from the gate.

Alphenaar et al..$^{77}$ have studied the scattering between edge channels in a double point-contact device similar to ours, but with a spacing of $80 \mu \mathrm{m}$ between the QPC's.
They find that in their device almost full equilibration of the edge channels takes place at $2.8 \mathrm{~T}$, with the noticeable exception of the upper edge channel. As a result, they observe an anomalous Hall conductance $G_{H}$ corresponding to $N_{L}-1$ Landau levels. McEuen et al. ${ }^{77}$ have explained their experimental results with a "decoupled network model," which explicitly takes into account the special role of the upper Landau level.

Finally we mention that edge channels can also be selectively populated or detected by using a $2 \mathrm{DEG}$ region in which the electron density is reduced by means of a gate on top of the heterostructure. ${ }^{78}$

\section{Anomalous quantization of the longitudinal resistance and adiabatic transport in series QPCS}

To calculate the longitudinal resistance $R_{L}$, which is defined as $R_{L}=\left(\mu_{6}-\mu_{4}\right) /(e I)$, with contacts 1 and 5 as current probes [Fig. 12(a)], we have to calculate the electrochemical potential of bulk contact 4 . Again we assume that this bulk contact is ideal, which gives the result $\mu_{4}=h /(2 e) I / N_{L}$. In a forward magnetic field the longitudinal resistance is given by

$$
R_{L}=\frac{\mu_{6}-\mu_{4}}{e I}=\left(\frac{1}{G_{H}}-\frac{h}{2 e^{2} N_{L}}\right)
$$

with $G_{I I}$ given by Eqs. (17)-(20). When $G_{H}$ and the bulk 2DEG are quantized, $R_{L}$ is also quantized at a value given by

$$
R_{L}=\frac{h}{2 e^{2}}\left(\frac{1}{\max \left(N_{A}, N_{B}\right)}-\frac{1}{N_{L}}\right) .
$$

In the regular QHE, the formation of a quantized plateau in $G_{H}$ is accompanied by the vanishing of the longitudinal resistance. This is because backscattering is absent in these magnetic-field ranges. ${ }^{6}$ The edge channels at a given boundary of the 2DEG are in mutual equilibrium, and all have the same electrochemical potential $\mu$. In this case the measured voltage is always $\mu / e$, independent of the details of the coupling of the voltage probes. The anomalous quantization of $R_{L}$ is a consequence of the nonequilibrium distribution created by QPC $A$. Because of the selective detection by QPC $B$, it measures a different electrochemical potential than bulk contact 4 , which measures the average electrochemical potential of the edge channels.

It should be noted that this mechanism for the anomalous quantization is different from the quantization that is observed when the longitudinal resistance is measured with probes located on either side of a region with a reduced electron density created by a cross gate ${ }^{79}$ or a split gate. ${ }^{80}$ In this case, the quantized longitudinal resistance arises from the backscattering of one or more edge channels, which is a result of the potential barrier created by the gated 2DEG region. This mechanism does not require the absence of scattering between adjacent edge channels.

Experimental results are given in Fig. 15, which gives a comparison between the two-terminal resistance $R_{B}=1 / G_{B}\left(R_{A}\right.$ and $R_{B}$ behave almost identically) and 


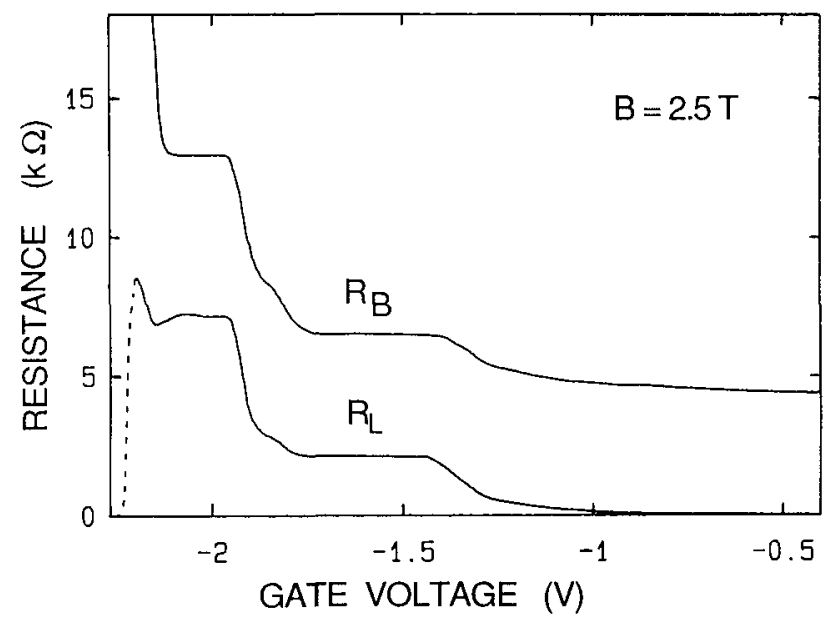

FIG. 15. Comparison between the two-terminal resistance $R_{B}$ of QPC $B$ and the longitudinal resistance $R_{L}$, showing anomalously quantized plateaus.

$R_{L}$. At $B=2.5 \mathrm{~T}$ the transport in the bulk 2DEG is quantized, with three occupied Landau levels. Plateaus are observed at $R_{L}=0\left[N_{L}=3\right.$ and $N_{A}, N_{B}=3$ in Eq. (22)], and at $R_{L}=\frac{1}{12}\left(h / e^{2}\right) \approx 2.11 \mathrm{k} \Omega \quad\left(N_{L}=3\right.$ and $\left.N_{A}, N_{B}=2\right)$. Although a precursor of the last plateau can be seen, it is not at its proper value of $\frac{1}{2}\left(h / e^{2}\right) \approx 8.6 \mathrm{k} \Omega$ $\left(N_{L}=3, N_{A}, N_{B}=1\right)$. This is probably due to the fact that inter-edge-channel scattering sets in when the QPC's are near pinchoff. At this magnetic field, no anomalous QHE is observed at low gate voltages either (see Fig. 13).

Transport through a series configuration of QPC's in the absence of a magnetic field has been studied experimentally by Wharam et al. ${ }^{81}$ and Main et al., ${ }^{82}$ and theoretically by Beenakker and van Houten. ${ }^{83}$ Kouwenhoven et al. ${ }^{84}$ studied the transition from the Ohmic transport regime in the absence of a magnetic field to the adiabatic transport regime in high magnetic fields. In this section we focus on the high-field regime where adiabatic transport in edge channels takes place. We study the two-terminal conductance $G_{S}$ measured between contacts 5 and 6 (the other contacts are not connected). The calculation proceeds along lines similar to those in Sec. IV C. Again we assume that the bulk contacts fully equilibrate the edge channels. The results are

$G_{S}=\frac{e I}{\mu_{5}-\mu_{6}}=\frac{2 e^{2}}{h}\left(N_{A}+T_{A}\right)$ when $N_{A}<N_{B}$,

$G_{S}=\frac{2 e^{2}}{h}\left(N_{B}+T_{B}\right)$ when $N_{A}>N_{B}$,

$G_{S}=\frac{2 e^{2}}{h} \frac{\left(N+T_{B}\right)\left(N+T_{A}\right)}{N+T_{A}+T_{B}-T_{A} T_{B}}$ when $N_{A}=N_{B}=N$.

Equations (23)-(25) state that $G_{S}$ is quantized when the QPC with the lowest conductance is quantized. The quantized value for $G_{S}$ is given by

$$
G_{S}=\frac{2 e^{2}}{h} \min \left(N_{A}, N_{B}\right) \text {. }
$$

This result can simply be understood by noting that the

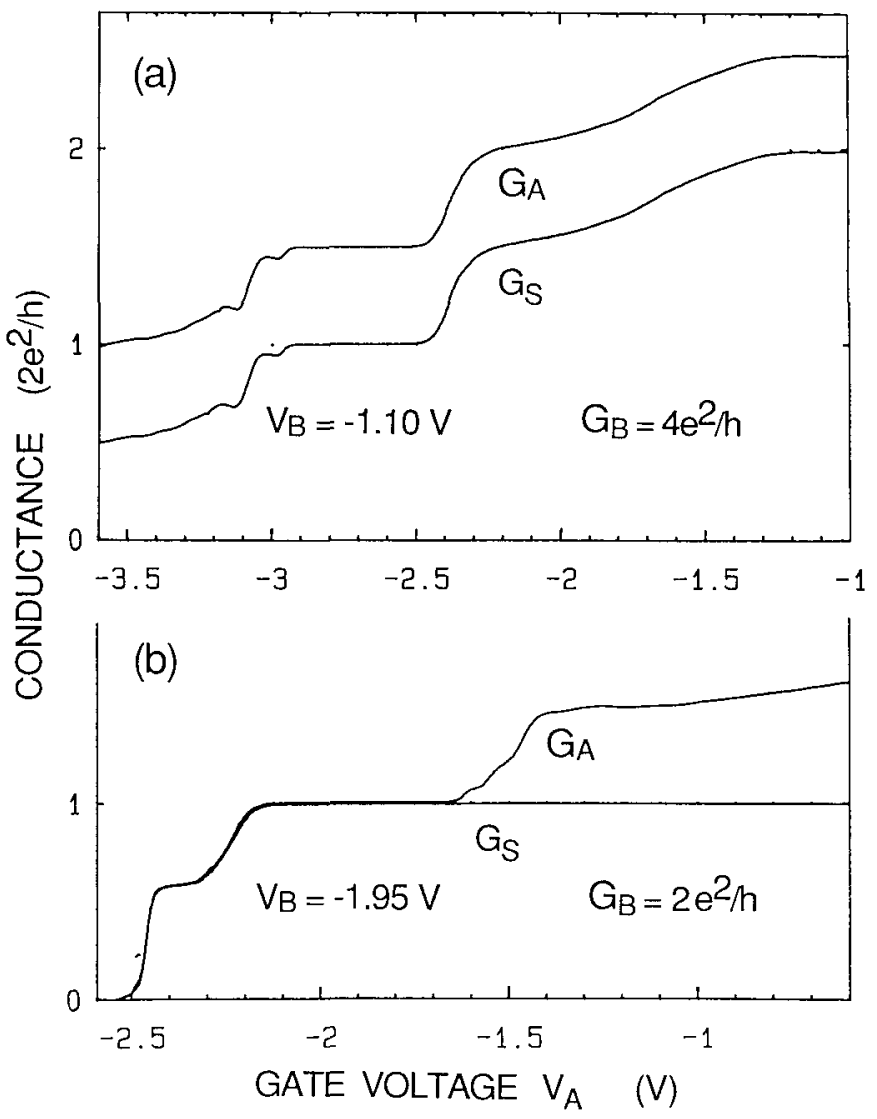

FIG. 16. Comparison between the two-terminal conductance $G_{A}$ of single QPC $A$ with the conductance $G_{S}$ of QPC's $A$ and $B$ in series (see text). The magnetic field is $3.3 \mathrm{~T}$. The upper trace in (a) has been shifted upwards by $e^{2} / h$ for clarity.

bottleneck for the transport is formed by the QPC with the highest potential barrier, which transmits the least number of edge channels. In contrast to the anomalous QHE, there is no difference when the magnetic field is reversed. This is because $G_{S}$ is a two-terminal conductance, which must be symmetric upon reversal of the magnetic field: ${ }^{69} \quad G_{S}(B)=G_{S}(-B)$. Note also that different expressions are obtained for a series configuration of QPC's without the presence of bulk contacts in the region between the QPC's. ${ }^{85}$ In this case there is no edge-channel equilibration in the region between the QPC's.

Figure 16(a) presents an experiment where $G_{B}$ was kept constant at $4 e^{2} / h$ and $G_{A}$ and $G_{S}$ were measured as a function of $V_{A}$. The number of occupied Landau levels $N_{L}=2$. In agreement with Eq. (23), $G_{S}$ is almost identical to $G_{A}$. In Fig. 16(b), QPC $B$ was fixed at $2 e^{2} / h$. Now $G_{S}$ closely follows $G_{A}$ when $G_{A}<2 e^{2} / h$, and is constant at $2 e^{2} / h$ when $G_{A}>2 e^{2} / h$. These results correspond with Eqs. (24) and (25), respectively.

\section{E. Inter- and intra-Landau-level scattering in high magnetic fields}

In this section we will include scattering in the model for electron transport. At low temperatures one expects elastic scattering to be dominant. This means that the 
appearance of a finite resistance (e $g$, the Shubnikov-de Haas oscillations) in a $2 D E G$ is not the consequence of dissipative piocesses In this respect there is no fundamental difference with the resistance of ballistic point contacts or the low-temperature residual resistance of metals The prime source of resistance is the elastic backscattering of the electrons Afterwards the electron distribution is equilibrated by inelastic processes, which, however, do not affect the resistance when the inelasticscattering rate is sufficiently weak

We therefore describe the scattering in terms of transmission probabilities $T$ and reflection probabilities $R$ between edge channels In our model we will distinguish between inter-Landau-level scattering and intra-Landaulevel scattering 8687

Inter-Landau-level scattering from one edge channel to another edge channel belonging to a different Landau level can occur at the edges of the $2 \mathrm{DEG}$, where the edge channels of different Landau levels are in close proximity (see F1g 10) Possible sources of inter-Landau-level scattering are impurities, irregularities of the $2 \mathrm{DEG}$ boundary, etc When the adjacent edge channels have the same electrochemical potential $\left[F_{1 g} 10(a)\right]$, there is no net scattering between them The edge channels are in equilibrium, and the scattering rate from one edge channel to another is perfectly compensated for by an equal scattering rate in the opposite direction Figure 10(b) 1llustrates that a net inter-Landau-level scattering rate can occur when two adjacent edge channels have a different electrochemical potential As shown in Sec IVC, such an inequilibrium occupation of adjacent edge channels can be created with QPC's It should be noted here that the scattering between adjacent edge channels does not reverse the direction of the current, and therefore does not produce backscattering

Intra-Landau-level scattering is the scattering from one edge channel to another edge channel belonging to the same Landau level, which flows in an opposite direction We will now show that the Shubnikov-de Haas $(\mathrm{SdH})$ oscillations, which are the most prominent manifestation of resistance in a $2 \mathrm{DEG}$, are the result of intra-Landau-level scattering of electrons in the upper Landau level Figure 10(a) 1llustrates the occupied electron states in a $2 \mathrm{DEG}$ for two occupred Landau levels The Fermi energy resides in between the flat parts of the Landau levels The electrons at the Fermi energy in the upper (second) Landau level flow along the edges of the $2 \mathrm{DEG}$ and follow equipotential lines at the edges of the $2 \mathrm{DEG}$ Because of the spatial separation of these edge channels, backscattering is absent and the Hall resistance is quantized When the magnetic field is increased, the bottom of the second Landau level approaches the Fermi energy It now becomes possible for the electrons in the second Landau level to scatter from one edge to another This will happen each time when the bottom of a Landau level crosses the Fermi energy, and this produces the Shubnikov-de Haas resistance oscillations Even though we do not know the exact nature of the scattering, we can nevertheless look upon the SdH oscillations as the backscattering of the electrons in the upper Landau level, distributed over the entire length of the $2 \mathrm{DEG}$ (a)

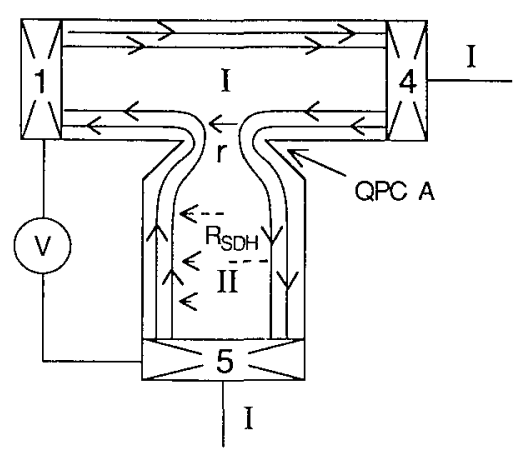

(b)

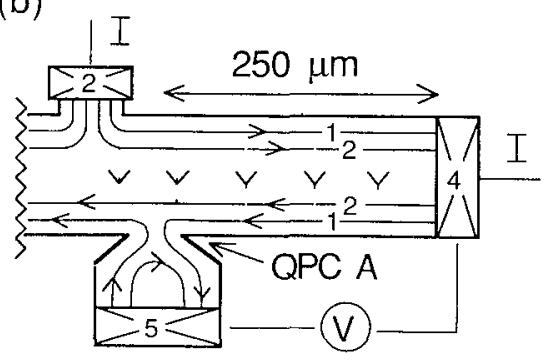

FIG 17 (a) Current flow in the presence of Shubnikov-de Haas backscattering, illustrating the mechanısm for suppression of the SdH oscillations due to selective population of edge channels by the QPC The reflection probability at the QPC is indicated by $r$ Dashed arrows illustrate the Shubnıkov-de Haas backscattering in region II, indicated by $R_{\mathrm{SdH}}$ (b) Mechanism for suppression of the $\mathrm{SdH}$ oscillations due to selective detection. of edge channels

We thus see that intra-Landau-level scattering is primarily due to the backscattering of electrons in the upper Landau level, and will be an oscillating function of the magnetıc field, being extremely weak at a quantum Hall plateau, and relatively strong at a Shubnikov-de Haas maximum The experiments presented in the following sections will show that the inter-Landau-level scattering can be extremely weak in high magnetic fields

We will now show that the magnitude of the SdH resistance depends on the transmission properties of the QPC's that serve as current or voltage probes $\mathrm{We}$ will discuss this for the geometry of Fig 17(a), which gives a simplified layout of the experimental geometry A threeterminal measurement is performed, with curient contacts 4 and 5 and voltage contacts 1 and 5 In the calculations we set $\mu_{5}=0$ The direction of the electron flow corresponds with a reverse magnetic field In this geometry we expect to measure a combination of the resistance of QPC $A$ and the SdH resistance of bulk $2 \mathrm{DEG}$ region II (the three-terminal setup reduces the effect of backscattering in 2DEG region I) Anticipating the experimental results, we assume that the only relevant source of scattering in the $2 \mathrm{DEG}$ is backscattering of electrons in the upper Landau level We also assume that we can use the edge-channel description for the upper Landau level, and also that the bulk contacts couple ideally to all $N_{L}$ edge channels (including the uppet edge channel, which is responsible for the $\mathrm{SdH}$ resistance) These assumptions make it possible to descirbe 
the total $\mathrm{SdH}$ backscattering in region II with a reflection probability $R_{\mathrm{SdH}}$.

The measured resistance $R_{45,15}$ is due to the backscattering of the electrons. This can happen at the QPC, where $N_{R}$ edge channels can be completely reflected, and one edge channel can be partially reffected, with reflection probability $r$ (see Sec. IV B). The second source of reflection is due to the $\mathrm{SdH}$ backscattering in the 2DEG region II. The combined reflection $R$ of both QPC and 2DEG region II can be obtained with the addition rule for reflection probabilities:

$$
R=\frac{r+R_{\mathrm{SdH}}-2 r R_{\mathrm{SdH}}}{1-r R_{\mathrm{SdH}}}
$$

when the QPC transmits the upper edge channel which is responsible for the $\mathrm{SdH}$ backscattering, and

$$
R=N_{R}+r
$$

when the QPC does not transmit the upper edge channel. The backscattered current is given by $I_{\text {back }}=(2 e / h) \mu_{4} R$, with $\mu_{4}$ the electrochemical potential of the electrons that enter the QPC from the right [see Fig. 17(a)]. The latter is given by $\mu_{4}=(h / 2 e) I /\left(N_{L}-R\right)$. The backscattered current flows towards contact 1 and builds up a voltage $V_{1}=\left(h / 2 e^{2}\right) / N_{L} I_{\text {back }}$. This gives for the measured resistance

$$
R_{45,15}=\frac{V_{1}}{I}=\frac{h}{2 e^{2}} \frac{R}{N_{L}\left(N_{L}-R\right)} .
$$

Two interesting conclusions can be drawn from this expression. First it shows that there is an upper bound on the magnitude of the SdH resistance. When the QPC transmits all edge channels completely $\left(N_{R}, r=0\right)$, the $\mathrm{SdH}$ resistance is limited to $R_{45,15}=\left(h / 2 e^{2}\right) 1 /\left[N_{L}\left(N_{L}\right.\right.$ -1 )] (an obvious exception is the case $N_{L}=1$, when the resistance can become infinite). This is an immediate consequence of the fact that the reflection probability $R_{\mathrm{SdH}}$ for the upper edge channel in region II cannot exceed 1. Because the number of occupied Landau levels $N_{L}$ is proportional to $1 / B$, Eq. (29) shows that the resistance at consecutive Shubnikov-de Haas maxima should be proportional to $B^{2}$, provided that the reflection probability $R_{\mathrm{SdH}}$ at the SdH maxima approaches unity. Although the maxima of the $\mathrm{SdH}$ resistance oscillations observed in a $2 D E G$ usually scale with $B$, rather than $B^{2}$, we believe that the mechanism for the increase is that the reflection probability $R_{\mathrm{SdH}}$ is not very much different for consecutive $\mathrm{SdH}$ maxima. The $\mathrm{SdH}$ resistance simply increases because the number of occupied Landau levels $N_{L}$ decreases with increasing field, as expressed in Eq. (29).

Another consequence of Eqs. (27)-(29) is that when the QPC does not transmit the upper Landau level, the measured resistance is given by $R_{45,15}=\left(N_{R}+r\right) /$ $\left[N_{L}\left(N_{L}-N_{R}-r\right)\right]$. This resistance is due to the complete or partial backscattering of edge channels at the QPC. The special thing about it is that it is independent of $R_{\mathrm{SdH}}$. This can be understood simply by the fact that the electrons in the upper edge channel are already completely reflected at the QPC, and the possible backscatter- ing in 2DEG region II becomes irrelevant. We conclude that the magnitude of the $\mathrm{SdH}$ oscillations will be suppressed when the QPC does not transmit the upper edge channel. A necessary condition is that the scattering between the upper edge channel and the low-lying edge channels (belonging to Landau levels with lower quantum numbers) is weak.

For forward-directed magnetic fields, the current flow in edge channels in reversed relative to Fig. 17(a). Electrons now approach the QPC from the left with electrochemical potential $\mu_{1}$. The measured resistance is now given by

$$
R_{45,15}=\frac{\mu_{1}}{e I}=\frac{h}{2 e^{2}} \frac{1}{N_{L}-R}
$$

with $R$ given by Eqs. (27) or (28). Similar to the situation in reverse field, we see that the resistance becomes independent of $R_{\mathrm{SdH}}$ when the QPC does not transmit the upper edge channel. Rewriting Eq. (30) with $R=N_{L}-\left(N_{A}+T_{A}\right)$ gives

$$
R_{45,15}=\frac{h}{2 e^{2}} \frac{1}{N_{A}+T_{A}} \text {. }
$$

When the upper edge channel is not transmitted by the QPC, the SdH oscillations are suppressed, and the measured resistance in forward fields is completely determined by the two-terminal resistance of the QPC.

We restrict ourselves to the above analysis of a threeterminal geometry. A similar suppression of the $\mathrm{SdH}$ resistance is expected to occur in the usual four-terminal geometry, when the longitudinal resistance is measured with two adjacent voltage probes. However, in this case the possibility of edge-channel mixing by the probes has to be taken into account (see Sec. IV H).

\section{F. Suppression of the Shubnikov - de Haas oscillations due to selective population and detection of edge channels}

In this section we present experimental results on the suppression of the $\mathrm{SdH}$ oscillations, predicted in the preceding section. The experimental setup corresponds with Fig. 17(a). Trace $a$ in Fig. 18 shows the results obtained at $V_{g}=-0.6 \mathrm{~V}$ in a reverse field. It is indicated which (single-spin) Landau levels are responsible for the $\mathrm{SdH}$ maxima. At $V_{g}=-0.6 \mathrm{~V}$, the QPC transmits all edge channels and a result $\mathrm{SdH}$ trace is observed, expected for this field orientation. Traces $b-e$ have been obtained in forward field. At $V_{g}=-0.6 \mathrm{~V}$, a superposition of the $\mathrm{SdH}$ oscillations and quantized plateaus is observed. When the gate voltage is reduced further, the position of the plateaus is determined by the QPC and they shift to lower fields. The residual structure on top of the quantized plateaus shows that the $\mathrm{SdH}$ oscillations are suppressed. Note the absence of the $N_{L}=3$ peak in trace $c$, and the suppression of the $N_{L}=4,6$, and 8 peaks in traces $d, e$, and $d$, respectively. These results correspond with Eq. (31), and they not only confirm that the SdH oscillations arise primarily from backscattering of the upper Landau level, but also that the majority of the electrons can flow from QPC $A$ to bulk contact 5, without 


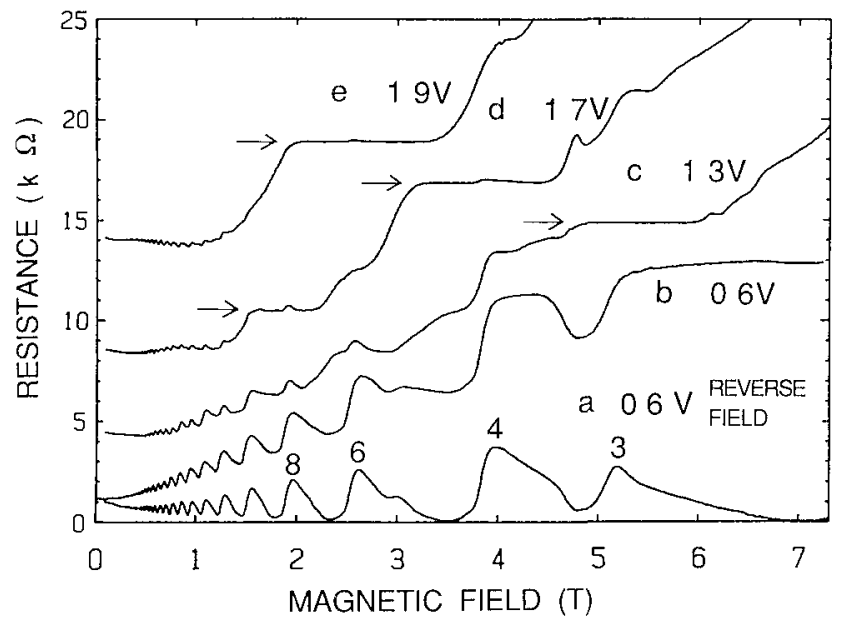

FIG 18 Suppression of the Shubnıkov-de Haas resistance oscillations due to selective population of edge channels Curve a shows regular $\mathrm{SdH}$ oscillations, measured in a reverse field Curves $b-e$ show the quantized resistance of the QPC in series with the SdH oscillations of the 2DEG Curves $c-e$ show suppressed $\mathrm{SdH}$ oscillations The arrows indicate the quantized values $h /\left(2 e^{2}\right)$ and $h /\left(4 e^{2}\right)$ The curves have been offset for clarity $c,+2 \mathrm{k} \Omega, d,+4 \mathrm{k} \Omega, e,+6 \mathrm{k} \Omega$

being scattered into the upper edge channel (electrons that are scattered into the upper Landau level will subsequently be scattered back and cause resistance) The results imply that the scattering between the upper edge channel and the low-lying edge channels is weak even on macroscopic length scales (Contact 5 is about $150 \mu \mathrm{m}$ away from QPC $A$ )

We return to trace $a$ According to the model description in the preceding section, the $\mathrm{SdH}$ maxima cannot exceed $\left(h / 2 e^{2}\right)\left[N_{L} / 2\left(N_{L} / 2-1\right)\right]^{1}$ when the spin degeneracy is not resolved and $h / e^{2}\left[N_{L}\left(N_{L}-1\right)\right]^{1}\left(N_{L}\right.$ indicates the number of occupied single-spin Landau levels) when the spin degeneracy is fully resolved A comparison with the experiment gives the following $N_{L}=3$, expt $27 \mathrm{k} \Omega$, theory (spin resolved) $43 \mathrm{k} \Omega, N_{L}=4$, expt $38 \mathrm{k} \Omega$, theory (spin resolved) $215 \mathrm{k} \Omega, N_{L}=6$, expt $25 \mathrm{k} \Omega$, theory (spin resolved) $870 \Omega$ This shows that the measured resistances exceed the theoretical $11 \mathrm{~m}$ its At present, this discrepancy is not understood It may be that there is some residual inter-edge-channel scattering in region II

To study the absence of scattering between adjacent edge channels further, we have performed a second experiment The configuration is given in $\mathrm{Fig}_{1 \mathrm{~g}}$ 17(b) We now expect to observe the suppression of the $\mathrm{SdH}$ oscillations due to the selective detection of edge channels This is 1llustrated for the case of two occupred Landau levels We set $\mu_{4}=0$ Contact 2 injects electrons into the two right-going edge channels As a result of the SdH backscattering, the second left-going edge channel acquires a nonzero electrochemical potential When QPC $A$ transmits all edge channels, a fintte voltage will be measured This voltage will vanish when the QPC does not couple to the upper edge channel, provided that there is no scattering between the upper edge channel and the low-lying edge channels in the region between bulk con-

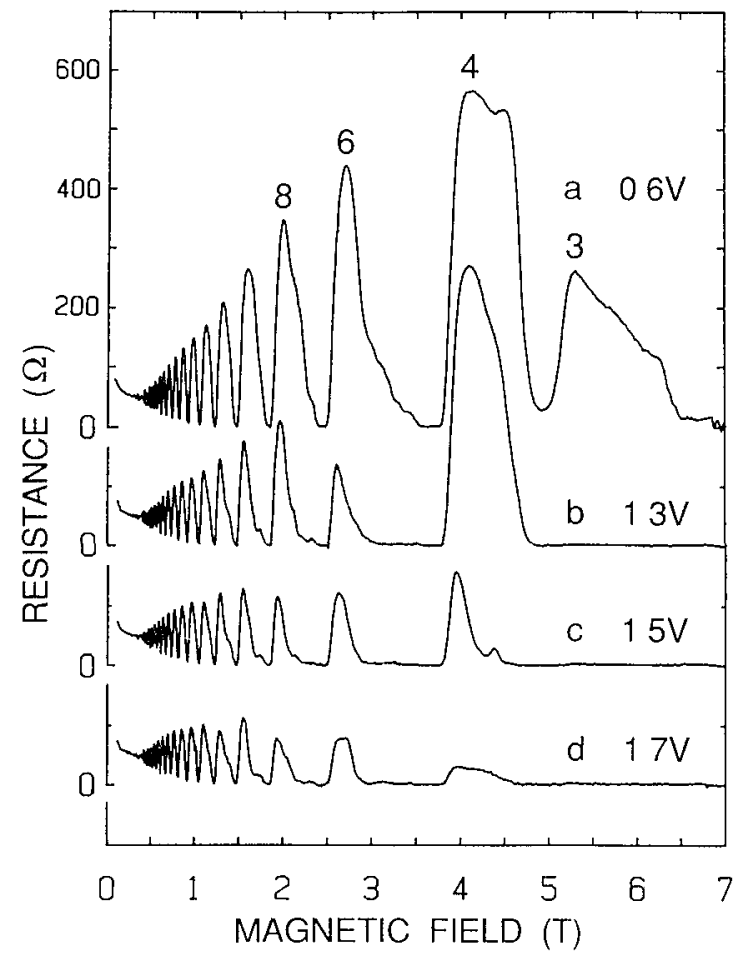

FIG 19 Suppression of the Shubnikov-de Haas resistance oscillations due to selective detection of edge channels The SdH oscillations are suppressed in curves $b, c$, and $d$

tact 4 and QPC $A$

Experimental iesults are given in Fig 19 At $V_{g}=-06 \mathrm{~V}$, the QPC transmits all edge channels and a more or less regular SdH trace is observed The fact that the $N_{L}=3$ and $N_{L}=5$ peaks are already partially suppressed is probably due to the fact that a small potential barrier is already present at this gate voltage When the gate voltage is reduced, the magnitude of the $\mathrm{SdH}$ peaks is substantially reduced At $V_{s}=-17 \mathrm{~V}$, the $N_{L}=3$ peak has almost disappeared (the residual iesistance is only a few ohms), and all other peaks above $10 \mathrm{~T}$ are substantially suppressed When we compare Figs 18 and 19 we see that the suppression of the $N_{L}=3$ max1mum in Fig 19 occurs at those magnetic fields where the QPC conductance is equal to, or lower than, $2 e^{2} / h\left(\mathrm{~F}_{1} \mathrm{~g}\right.$ 18), which means that the QPC does not transmit the third edge channel This shows that the $\mathrm{SdH}$ resistance is suppressed when the QPC does not transmit the upper edge channel The suppression of the $\mathrm{SdH}$ oscillations shows that in the region between bulk contact 4 and the QPC, only very little scattering occurs between the upper and the low-lying edge channels 707778 As discussed in Sec IV C, a possible explanation may be that the scattering is suppressed because of reduced overlap of the wave functions of the different edge channels The low-lying edge channels follow equipotential lines near the edge of the 2DEG, whereas the upper Landau level (which follows the lowest equipotentral line) may be located away from the $2 D E G$ boundary, and may possibly follow a percolating path through the inten or of the 2DEG Howevel, the experiments show that the $\mathrm{SdH}$ resistance is not suppressed at $V_{s}=-06 \mathrm{~V}$, when the QPC is about 250 
$\mathrm{nm}$ wide. This means that the upper edge channel cannot be further away from the edge than about $250 \mathrm{~nm}$.

We emphasize that the observed suppression of the $\mathrm{SdH}$ oscillations does not necessarily mean that the scattering between a pair of low-lying edge channels is weak on macroscopic length scales. In fact, because the upper edge channel may be located relatively far away from the 2DEG boundary, it may be possible that the scattering rate between the upper edge channel and the low-lying edge channels is different from the scattering rate between a pair of low-lying edge channels. We can investigate this experimentally by observing the suppression of the $N_{L}=8 \mathrm{SdH}$ maximum at $B=2.0 \mathrm{~T}$ in Fig. 19 . At $V_{g}=-1.3 \mathrm{~V}$, QPC $A$ shows a plateau at $h /\left(6 e^{2}\right)$ (Fig. 18), which means that it only transmits six (singlespin) edge channels, and does not transmit the edge channel that is responsible for the $\mathrm{SdH}$ backscattering anymore. As expected, the $N_{L}=8$ peak in Fig. 19 is partially suppressed. However, when the gate voltage is reduced, the SdH peak is suppressed further. At $V_{g}=-1.7 \mathrm{~V}$, QPC $A$ only transmits four edge channels (Fig. 18). The fact that the measured resistance depends on the number of transmitted edge channels implies that the low-lying edge channels are not in equilibrium, and are occupied up to different electrochemical potentials. To be precise, the edge channels with the lowest Landaulevel indices have the lowest electrochemical potential. Although it is difficult to give a quantitative analysis, this lack of equilibration between the low-lying edge channels means that the scattering between the low-lying edge channels is also weak. Recent experiments ${ }^{77,78}$ show that equilibration lengths between low-lying edge channels are typically $20-40 \mu \mathrm{m}$.

The experiments show that at the $\mathrm{SdH}$ maximum at 5.2 $\mathrm{T}$ the first two edge channels that arrive at the QPC are almost completely empty. This does not only mean that the scattering of electrons into these edge channels is (almost) zero in the 2DEG itself, but also that no (partial) backscattering of these edge channels occurs at contact 4 . This shows that at the SdH maximum due to backscattering of electrons in the third edge channel, contact 4 still couples ideally to the first two edge channels.

\section{G. Edge-channel mixing controlled by quantum point contacts}

An important feature of nonlocal transport is that the voltage measured with a particular voltage probe can be affected by the presence of other voltage probes. When QPC's are used as probes, the mechanism is as follows [see Fig. 20(a)]: Unequally populated edge channels that enter QPC $B$ will finally reach bulk contact 6 and equilibrate. The electrochemical potential of these edge channels will therefore be different when they leave QPC $B$. This change in edge-channel occupation will affect the voltage measured with the subsequent QPC $A$, provided that this QPC couples selectively to the edge channels. Figure 20(a) illustrates the situation for the case of three (single-spin) edge channels (for clarity, the first two edge channels have been drawn as one). The third edge channel is populated $\left(\mu_{3} \neq 0\right)$ and the other two are empty
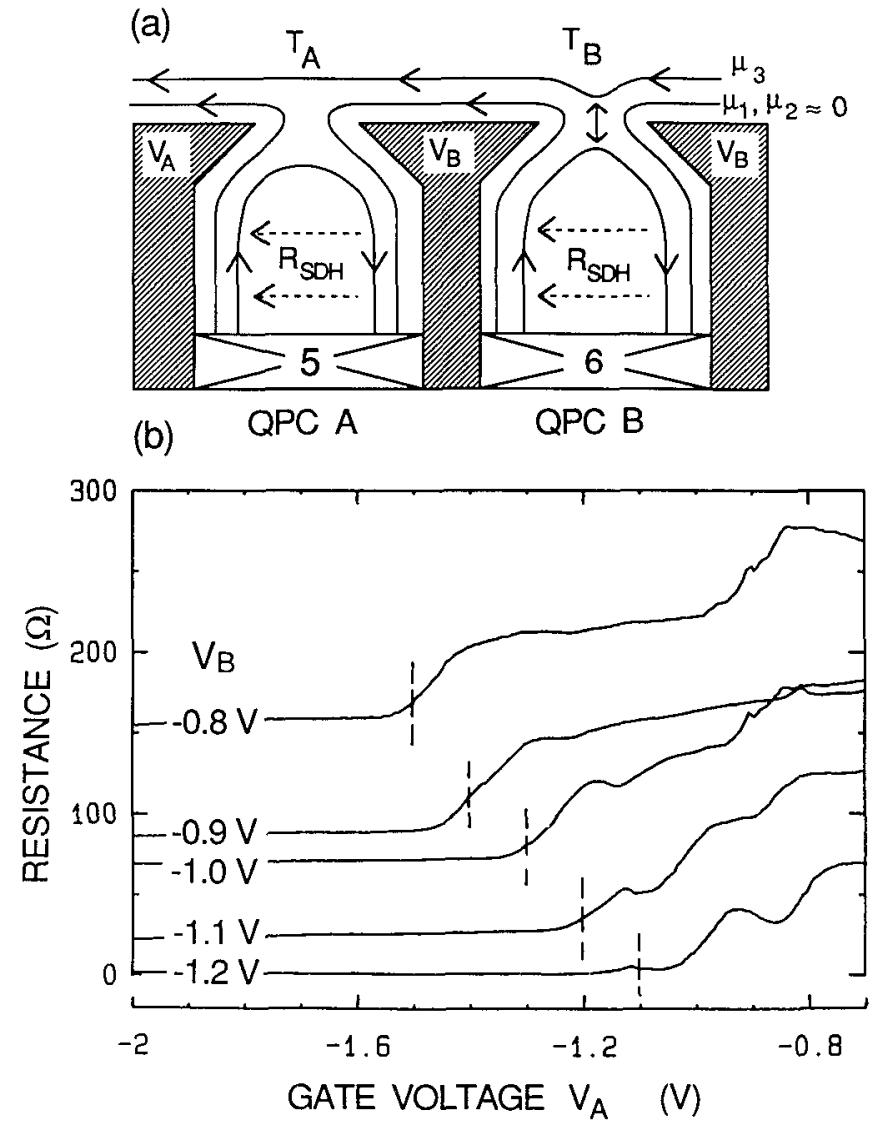

FIG. 20. (a) Current flow in edge channels, illustrated for the case of three occupied single-spin Landau levels. For clarity the first two edge channels have been drawn as one. (b) Resistance measured between contacts 5 and 4 , with 2 and 4 as current contacts, measured as a function of gate voltage $V_{A}$ for several fixed values of the gate voltage $V_{B}$. The vertical dashed lines indicate where the effective gate voltage $\left(V_{A}+V_{B}\right) / 2$ is equal to -1.15 V.

$\left(\mu_{1}, \mu_{2}=0\right)$. We will now calculate how $\mu_{5}$ depends on the transmission properties of both QPC $A$ and $B$. The electrochemical potential $\mu_{4}=0$ in the calculation (see Fig. 1). First we note that the third edge channel can be reflected at the QPC's themselves (with probabilities $r_{A}$ and $r_{B}$ ), and also in the 2DEG regions behind the QPC's, as a result of the $\mathrm{SdH}$ backscattering. This gives a reflection probability $R_{\mathrm{SdH}}$, which we assume equal for both QPC's [the 2DEG regions behind both QPC's have equal dimensions (see Fig. 1)]. It was shown in Sec. IV E [Eq. (27)] that both sources of reflection can be combined to give a total reflection $R_{A}$ and $R_{B}$ for the third edge channel at probes $A$ and $B$ :

$$
R_{A / B}=\frac{r_{A / B}+R_{\mathrm{SdH}}-2 r_{A / B} R_{\mathrm{SdH}}}{1-r_{A / B} R_{\mathrm{SdH}}} .
$$

First we consider the situation where the third edge channel is not transmitted through QPC $B$. This means that QPC $B$ does not alter the occupation of the edge channels. (Only edge channels 1 and 2 are transmitted; they are initially empty and will remain so when they leave QPC $B$.) The voltage measured with QPC $A$ is now 


$$
V_{5}=\frac{\mu_{3}}{e} \frac{T_{A}}{2+T_{A}} \text { with } T_{A}=1-R_{A}
$$

when QPC $A$ transmits the third edge channel and

$$
V_{5}=0
$$

when QPC $A$ does not transmit the third edge channel Up to now the situation is not different from the suppression of the SdH resistance due to the selective detection of edge channels, discussed in the preceding section However, the situation changes when QPC $B$ starts transmitting the third edge channel The electrochemical potential of QPC $B$ now becomes

$$
\mu_{6}=\frac{T_{B}}{2+T_{B}} \mu_{3} \text { with } T_{B}=1-R_{B}
$$

Now the first and second edge channels that leave QPC $B$ are not empty anymore, but carry a current $I_{1}+I_{2}=(e / h) \mu_{3} 2 T_{B} /\left(2+T_{B}\right)$ The third edge channel carries a current $I_{3}=(e / h) \mu_{3}\left[T_{B}^{2} /\left(2+T_{B}\right)+\left(1-T_{B}\right)\right]$ To calculate the voltage at QPC $A$, we distınguish between two situations, depending on whether the third edge channel is transmitted by QPC $A$ or not In the former case we obtain

$$
V_{5}=\frac{\mu_{3}}{e} \frac{2 T_{A}+2 T_{B}-T_{A} T_{B}}{\left(2+T_{A}\right)\left(2+T_{B}\right)} \text { when } T_{A} \neq 0
$$

whereas the latter case gives

$$
V_{5}=\frac{\mu_{6}}{e}=\frac{\mu_{3}}{e} \frac{T_{B}}{2+T_{B}} \quad \text { when } T_{A}=0
$$

We see that when QPC $B$ (partially) transmits the third edge channel, $V_{5}$ does not become zero anymore when the QPC $A$ couples to the (initially empty) first and second edge channels only $\left(T_{A}=0\right)$, but saturates at a constant value, determined by QPC $B[\mathrm{Eq}$ (37)] This shows that the presence of a voltage probe can create a finite resistance

We have performed an experiment $\left[\begin{array}{ll}F_{1} g & 20(b)\end{array}\right]$ in which we have created an unequal occupation of edge channels by tuning the magnetic field at the Shubnikov-de Haas maxımum at $B=52 \mathrm{~T}$ Contacts 2 and 4 are cur rent contacts, and 4 and 5 are voltage contacts The analysis in the preceding section shows that because of the absence of scattering between edge chan nels, the first two edge channels arrive almost empty at QPC $B$, and only the third edge channel is occupied In the experiment the transmission through QPC $B$ was kept fixed at several fixed values of $V_{B}$, and the tiansmission thiough QPC $A$ was varied by changing $V_{A}$ [note that the effective gate voltage that defines QPC $A$ is approximately given by $\left(V_{1}+V_{B}\right) / 2$ ] From measurements of the two-terminal conductance of the QPC's, it was found that the third edge channel is transmitted at gate voltages of $-115 \mathrm{~V}$ and higher The bottom curve in Fig 20(b) shows the result when $V_{B}<-115 \mathrm{~V}$, and QPC $B$ does not transmit the third edge channel For $V_{A}>-115 \mathrm{~V}$, a resistance is measured, and for $V_{A}<-115 \mathrm{~V}$, when the third edge channel is not transmitted anymore by QPC $A$, the resistance vanishes, in agreement with Eqs (33) and (34) The situation changes at gate voltages $V_{B}>-115 \mathrm{~V}$ The resistance does not vanish anymore, but saturates at a constant value when QPC $A$ couples to the first and second edge channel only (the vertical dashed lines approximately indicate the threshold values where the effective gate voltage is $-115 \mathrm{~V}$ ) When the transmission $T_{B}$ is increased, the resistance at the plateau also increases, which corresponds with $\mathrm{Eq}$ (37) These results are the experimental proof that QPC $B$ together with bulk contact 6 acts as a controllable "edge-channel mixer"

Measurements of the two-terminal conductances of the QPC's show that their conductances are approximately equal when $V_{A}=V_{B}$ We now make a comparison between the resistance measured in this case, which corresponds to $\mathrm{Eq}$ (36) with $T_{A}=T_{B}=T$, and the resistance measured with the same voltage on $V_{B}$ only and a gate voltage on gate $A$, such that QPC $A$ does not transmit the third edge channel [this case is given by Eq (37)] The ratio of the two voltages given by Eqs (36) and (37) is given by $(4-T) /(2+T)$

The experimentally observed ratios are $-08 \mathrm{~V}, 175$, $-09 \mathrm{~V}, 20,-10 \mathrm{~V}, 19$, and $-11 \mathrm{~V}, 20$ It was concluded fiom the analysis of the SdH oscillations that the total reflection probabilities $R_{A}$ and $R_{B}$ at a $\mathrm{SdH}$ maxImum are near unity, and the corresponding $T_{A}$ and $T_{B}$ are small At low gate voltages the expenimental values are therefore in excellent agreement with the theoretical ratio of 2 , expected for low transmissions The agreement between the experiments and our model indicates that a description of the electron transport in terms of edge channels remains valıd even at a maximum of the $\mathrm{SdH}$ resistance

We can now make a rough est1mate for $\mu_{3}$, the electrochemical potentral of the third edge channel at the entrance of QPC $B$ From the ratıo 175 at $V_{g}=-08 \mathrm{~V}$, we find $T \approx 02$ From the measured resistance at the plateau $(160 \Omega)$ we find, with $\mathrm{Eq}(37), \mu_{3} /(e I) \approx 11 \mathrm{k} \Omega$ This has to be compared with the electrochemical potentral difference $\mu$ between the cur rent contacts 2 and 4 , which is limited by $h /(2 e)>\mu / I>h /(3 e)$ This means that the electrochemical potential of the third edge channel is a considerable fraction of the total electrochemical potential across the sample This means that the scattering at a SdH maximum is strong

The SdH scattering rate can be measured directly in a Corbino geometiy 8889 Experiments on Corbino disks show that when the 2DEG is quantized, the resistance between interior and exterior edges of the 2DEG becomes extremely high This is because of the absence of Shubnikov-de Haas scattering between the contacts At magnetic fields that correspond with a maximum of the Shubnikov-de Haas scattering rate, which in the case of a Corbino geometry implies a maximum in the transmission between the interior and exterior contact, the sheet lesistance of the $2 \mathrm{DEG}$ is about $10-100 \mathrm{k} \Omega$ Compared to the resistance of a single edge channel $(258 \mathrm{k} \Omega)$, this again confirms that the $\mathrm{SdH}$ scattering is strong 


\section{H. Conclusions and discussion}

From our experiments, the following picture for highmagnetic-field transport emerges The electron transport is almost perfectly adiabatic on length scales of the order of several $\mu \mathrm{m}$, and may even be (partially) adiabatic on length scales exceeding $200 \mu \mathrm{m}$ for the case of the upper Landau level The electrons flow in edge channels, with only little change of being scattered into other edge channels The major scattering processes occur at the Shubnikov-de Haas maxima, when electrons in the upper Landau level can be scattered to the opposite edge of the $2 \mathrm{DEG}$ The transport in the low-lying Landau levels can be described completely in terms of edge channels, which are located at the boundary of the $2 \mathrm{DEG}$ The experiments seem to indicate that an edge-channel description works for the upper Landau level as well, even at a Shubnikov-de Haas maximum, where the scatterıng is severe However, at these $\mathrm{SdH}$ maxima the electrons in the upper Landau level are not bound to the edges anymore, but can move throughout the interior of the $2 \mathrm{DEG}$ A further study is required to explain how the edge-channel description for the electron transport in the upper Landau level can be reconciled with the pinning of the Fermı level to the upper Landau level in the bulk of the 2DEG Also, the detalled mechanism of the Shubnikov-de Haas backscatterıng, and together with it the appearance of the quantum Hall plateaus that occur when the $\mathrm{SdH}$ backscattering is absent, remain to be explained For a complete picture, the (localized) states in the bulk $2 \mathrm{DEG}$ may have to be taken into account
Because of the lack of equilibration in the 2DEG itself, our experiments show that in micrometer scale devices the accuracy of the QHE depends crucially on the ideal coupling of the contacts As shown by Buttıker, ${ }^{6}$ at least two adjacent ideal contacts are required to obtain the QHE In larger devices (of the order of $100 \mu \mathrm{m}$ ), the contacts may influence the $\mathrm{QHE}$

A first requirement for ideal contacts is that the electron density in the 2DEG near the contact must be equal or higher than the electron density of the bulk 2DEG to avold backscattering of one or more edge channels at the contact Our experiments have shown that this is generally the case ${ }^{90}$ Because the mixing of edge channels in the $2 \mathrm{DEG}$ itself is weak, we believe that the actual mixing occurs in a region of the contact where the twodimensional electron gas is completely destroyed Considering their important role in the transport in high magnetic fields, a further study of the physics of contacts is desirable

\section{ACKNOWLEDGMENTS}

The authors thank D van der Marel, E Haanappel, L W Molenkamp, L I Glazman, A D Stone, R G Wheeler, B W Alphenaar, and P L McEuen for fruitful discussions, C E Timmering and $\mathrm{L} W$ Lander for technological support, J J Harris for the supply of the highmobility material, the Delft Centre for Submicron Technology for the facilities offered, and the Stichting voor Fundamenteel Onderzoek der Materie (FOM) for financral support
${ }^{\mathrm{I}} \mathrm{T} \mathrm{J}$ Thornton, $\mathrm{M}$ Pepper, $\mathrm{H}$ Ahmed, $\mathrm{D}$ Andrews, and $\mathrm{G} \mathbf{J}$ Davies, Phys Rev Lett 56, 1198 (1986)

${ }^{2} \mathrm{H} \mathrm{Z}$ Zheng, H P Wer, D C Tsui, and G Wermann, Phys Rev B 34, 5635 (1986)

${ }^{3}$ For reviews of quantum ballistic transport in QPC's see $\mathrm{H}$ van Houten, C W J Beenakker, and B J van Wees, in Semiconductors and Semimetals, edited by $\mathrm{M}$ A Reed (Academic, New York, in press), C W J Beenakker and $\mathrm{H}$ van Houten, in Solld State Physics, edited by D Turnbull and $\mathrm{H}$ Ehrenreich (Academic, New York, in press)

${ }^{4} \mathrm{~K}$ von Klitzing, G Dorda, and $M$ Pepper, Phys Rev Lett 45, 494 (1980)

${ }^{5}$ The Quantum Hall Effect, edited by R E Prange and S M Girvin (Springer-Verlag, New York, 1987)

${ }^{6} \mathrm{M}$ Buttıker, Phys Rev B 38, 9375 (1988)

${ }^{7}$ P Streda, J Kucera, and A H MacDonald, Phys Rev Lett 59, 1973 (1987), J K Jain and S A Kivelson, ıbıd 60, 1542 (1988)

${ }^{8}$ B I Halperın, Phys Rev B 25, 2185 (1982)

${ }^{9}$ B J van Wees, E M M Willems, C J P M Harmans, C W J Beenakker, $\mathrm{H}$ van Houten, $\mathbf{J} \mathrm{G}$ Willamson, $\mathrm{C} \mathrm{T}$ Foxon, and J J Harrıs, Phys Rev Lett 62, 1181 (1989)

${ }^{10} \mathrm{~B} \mathrm{~J}$ van Wees, E M M Willems, L P Kouwenhoven, C J P M Harmans, J G Willamson, C $T$ Foxon, and $J$ Harris, Phys Rev B 39, 8066 (1989)

${ }^{11} \mathrm{~B} J$ van Wees, $\mathrm{H}$ van Houten, C W J Beenakker, J G Williamson, L P Kouwenhoven, $D$ van der Marel, and $C \mathrm{~T}$ Foxon, Phy Rev Lett 60, 848 (1988)
${ }^{12} \mathrm{~B} \mathrm{~J}$ van Wees, L P Kouwenhoven, $\mathrm{H}$ van Houten, $\mathrm{C}$ W J Beenakker, J E Moorj, C T Foxon, and J J Harris, Phys Rev B 38, 3625 (1988)

${ }^{13} \mathrm{H}$ van Houten, B J van Wees, J E Moo1j, C W J Beenakker, J G Williamson, and C T Foxon, Europhys Lett 5, 721 (1988)

${ }^{14} \mathrm{C}$ W J Beenakker, $\mathrm{H}$ van Houten, and B J van Wees, Europhys Lett 7, 359 (1988)

${ }^{15} \mathrm{H}$ van Houten, $\mathrm{C}$ W J Beenakker, J G Willamson, $\mathrm{M}$ E I Broekaart, P H M van Loosdrecht, B J van Wees, J E Mooy, C T Foxon, and J J Harrıs, Phys Rev B 39, 8556 (1989)

16J G Willamson, H van Houten, C W J Beenakker, M E I Broekaart, L I A Spendeler, B J van Wees, and C $\mathrm{T}$ Foxon, Phys Rev B 41, 1207 (1990)

${ }^{17} \mathrm{~L}$ P Kouwenhoven, B J van Wees, C J P M Harmans, J $G$ Willamson, $H$ van Houten, $C$ W J Beenakker, C $T$ Foxon, and J J Harris, Phys Rev B 39, 8040 (1989)

${ }^{18} \mathrm{P}$ H M van Loosdrecht, C W J Beenakker, $\mathbf{H}$ van Houten, J G Willamson, B J van Wees, J E Moo1j, C T Foxon, and J J Harris, Phys Rev B 38, 10162 (1988)

${ }^{19}$ Point contacts in a $2 \mathrm{DEG}$, fabricated with etching technıques, were first used by $\mathrm{J} R$ Kirtley, $\mathrm{Z}$ Schlesinger, $\mathrm{T} \mathrm{N}$ The1s, $\mathrm{F}$ P Millken, S L Wright, and L F Palmateer, Phys Rev B 34, 5414 (1986)

${ }^{20} \mathrm{R}$ Landauer, IBM J Res Dev 1, 223 (1957), Phys Lett 85A, $91(1981)$

${ }^{21} \mathrm{Yu}$ V Sharvin, Zh Eksp Teor F1z 48, 984 (1965) [Sov 
Phys - JETP 21, 655 (1965)]

${ }^{22}$ Metallic point contacts have been used extensively for the study of Fermi surfaces, electron-phonon interaction, etc For a review, see A G $M$ Jansen, A P van Gelder, and P Wyder, J Phvs C 13, 6073 (1980)

${ }^{23}$ This three-terminal measurement eliminates a possible series resistance from $2 \mathrm{DEG}$ region I, and measures the QPC resistance in series with resistance of 2 DEG region II and a possible resistance of bulk contact 5

${ }^{24} \mathrm{D}$ A Wharam, T J Thornton, R Newbury, M Pepper, $\mathrm{H}$ Ahmed, J E F Frost, D G Hasko, D C Peacock, D A Ritchie, and G A C Jones, J Phys C 21, L209 (1988)

${ }^{25}$ Conductance quantization has also been observed in devices fabricated by focused ion beam lithography Y Hirayama and T Saku, Appl Phys Lett 54, 2556 (1989), and by wet chemical etching Y Takagakı et al (unpublished)

${ }^{26}$ The gate voltage changes slightly the width and the resistance of 2 DEG region $\mathrm{II}$, which leads to the point contact The estimated effect is about $10 \Omega / \mathrm{V}$

${ }^{27} \mathrm{~A}$ detalled study of the accuracy of the point-contact quant1zation has been performed by $G$ Timp, $R$ Behrıngeı, S Sampere, J E Cunningham, and R E Howard, in Proceedings of the International Symposium on Nanostructure Physics and Fabrication, edited by W P Kirk and M Reed (Academic, New York, 1989)

${ }^{28}$ S E Laux, D J Frank, and F Stern, Surf Scı 196, 101 (1988)

${ }^{29} \mathrm{Y}$ Imry, in Directions in Condensed Matter Physics, edited by $G$ Grinstein and $G$ Mazenko (World Scientıfic, Singapore, 1986), Vol 1, p 102

${ }^{30}$ Several authors have obtained transmission resonances in the calculated conductance of narrow constrictions, see Refs $31-60$

${ }^{31}$ P F Bagwell and T P Orlando, Phys Rev B 40, 1456 (1989)

${ }^{32} \mathrm{U}$ Sivan, $M$ Heiblum, and $C P$ Umbach, Phys Rev Lett 63, 992 (1989)

${ }^{33}$ From measurements of the slope of the plateaus as a function of temperature, 11 is possible to extract the subband spacing $\hbar \omega_{0}$ The obtained values correspond well with the values obtained from the analysis of the magnetoconductance in $\mathrm{Sec}$ III F

${ }^{34} \mathrm{Nixon}$ et al (unpublished), Laughton et al (unpublished)

${ }^{35}$ A Szafer and A D Stone, Phys Rev Lett 62, 300 (1989)

${ }^{36} \mathrm{E} G$ Hadnappel and D van der Marel, Phys Rev B 39, 5484 (1989), D van der Marel and E G Haanappel, tbıd 39, 7811 (1989)

${ }^{37} \mathrm{~A}$ Yacoby and Y Imry (unpublished)

${ }^{38} \mathrm{G}$ Kirczenow, Solıd State Commun 68, 715 (1988), Phys Rev B 39, 10452 (1989)

${ }^{39} \mathrm{E}$ Tekman and S Ciracı, Phys Rev B 39, 8772 (1989), 42, 9098 (1990), 40, 8559 (1989)

${ }^{40} \mathrm{~L}$ I Glazman and A V Khaetskı1, Europhys Lett 9, 263 (1989)

${ }^{41}$ L I Glazman, G B Lesovik, D E Khmel'nitski1, and R I Shekhter, P1s'ma Zh Eksp Teor Fiz 48, 218 (1988) [JETP Lett 48, 238 (1988)], L I Glazman and M Jonson, Phys Rev B 41, 10686 (1990)

${ }^{42} \mathrm{~L}$ I Glazman and A V Khaetskil, Pis'ma Zh Eksp Teor F1z 48, 546 (1988) [JETP Lett 48, 591 (1988)]

${ }^{43}$ L Escapa and N Garcia, J Phys Condens Matter 1, 2125 (1989)

${ }^{44}$ A Kawabata, J Phys Soc Jpn 58, 372 (1989)

${ }^{45}$ A Matulis and D Segzda, J Phys Condens Matter 1, 2289 (1989)
${ }^{46} \mathrm{R}$ Johnston and L Schweitzer, J Phys C 21, L861 (1988)

${ }^{47} \mathrm{Y}$ Isawa and $\mathrm{Y}$ Isawa (unpublished)

${ }^{48} \mathrm{~J}$ Masek and B Kramer (unpublished)

${ }^{49}$ B Kramer and J Masek, J Phys C 21, L1147 (1988)

${ }^{50}$ Song He and S Das Sarma, Phys Rev B 40, 3379 (1989)

${ }^{51} \mathrm{E}$ G Castano and G Kirzenow, Solid State Commun 70, 801 (1989)

${ }^{52} \mathrm{~N}$ Garcia and L Escapa, Appl Phys Lett 54, 1418 (1989)

${ }^{53}$ I B Levinson, Pis'ma Zh Eksp Teor Fiz 48, 273 (1988) [JETP Lett 48, 301 (1988)]

${ }^{54} \mathrm{Y}$ Avishal and Y B Band, Phys Rev B 40, 3429 (1989), 40, 12535 (1989)

${ }^{55} \mathrm{G}$ Kirzenow, Phys Rev B 38, 10598 (1988)

${ }^{56} \mathrm{P}$ E Lindelof, Phys Lett A 132, 209 (1988)

${ }^{57}$ A Widom and R Tao, J Phys C 21, L1061 (1988)

${ }^{58} \mathrm{~K}$ B Efetov (unpublished)

${ }^{59}$ M Buttiker, Phys Rev B 41, 7906 (1990)

${ }^{60} \mathrm{~V}$ B Shikin, Pis'ma Zh Eksp Teor Fiz 50, 150 (1989) [JETP Lett 50, 167 (1989)]

${ }^{61} \mathrm{~K}$ F Berggren, $\mathrm{T}$ J Thornton, D J Newson, and M Pepper, Phys Rev Lett 57, 1769 (1986), S B Kaplan and A C Warren, Phys Rev B 34, 1346 (1986)

${ }^{62}$ We ignore the effect of the oscillations of the bulk Fermı energy with magnetic field, which may result from the pinning of the Fermi energy to the upper Landau level

${ }^{63} \mathrm{G}$ Kirzenow, Phys Rev B 38, 10958 (1988)

${ }^{64}$ J F Weisz and K F Berggren, Phys Rev B 40, 1325 (1989)

${ }^{65} \mathrm{D}$ A Wharam, U Ekenberg, M Pepper, D G Hasko, H Ahmed, J E F Frost, D A Ritchie, D C Peacock, and G A C Jones, Phys Rev B 39, 6283 (1989)

${ }^{66} \mathrm{U}$ Wulf, V Gudmunsson, and R R Gerhardts, Phys Rev B 38, $4218(1988)$

${ }^{67}$ A L Efros, Solid State Commun 67, 1019 (1988)

${ }^{68}$ The transmission of edge channels through a region of reduced electron density has been studied theoretically by $\mathrm{H}$ A Fertig and B I Halperın, Phys Rev B 36, 7969 (1987), T Ohtsukı and $\mathrm{Y}$ Ono (unpublished)

${ }^{69} \mathrm{M}$ Buttıker, Phys Rev Lett 57, 1761 (1986), IBM J Res Dev 32, 317 (1988)

${ }^{70}$ S Komiyama, H Hiral, S Sasa, and S Hiyamizu, Phys Rev B 40, 12566 (1989), Soltd State Commun 73, 91 (1990), S Komiyama and H Hira1, Phys Rev B 40, 7767 (1989)

${ }^{71}$ U Sivan, Y Imry, and C Hartzstem, Phys Rev B 39, 1242 (1989)

${ }^{72} \mathrm{~A}$ M Chang and $\mathbf{J}$ E Cunningham, Solid State Commun 72, 651 (1989), L P Kouwenhoven et al, Phys Rev Lett 64, 685 (1990)

${ }^{73} \mathrm{C}$ W J Beenakke1, Phys Rev Lett 64, 216 (1990), A H MacDonald, sbid 64, 220 (1990)

${ }^{74} \mathrm{~L}$ I Glazman and $\mathrm{M}$ Jonson, J Phys Condens Matter 1, 5547 (1989), Phys Rev Lett 64, 1186 (1990)

${ }^{75} \mathrm{~T}$ Martın and S Feng, Phys Rev Lett 64, 1971 (1990)

${ }^{76} \mathrm{~J} \mathrm{~J}$ Palacios and C Tejedor (unpublished)

${ }^{77} \mathrm{~B}$ W Alphenaar et al, Phys Rev Lett 64, 677 (1990), P L McEuen et al, lbtd 64, 2062 (1990)

${ }^{78} \mathrm{G}$ Muller et al, Phys Rev B 42, 7633 (1990)

${ }^{79} \mathrm{~S}$ Washburn, A B Fowler, H Schm1d, and D Kern, Phys Rev Lett 61, 2801 (1988), R J Haug, A H MacDonald, P Streda, and K von Klitzıng, tbıld 61, 2797 (1988)

${ }^{80} \mathrm{H}$ van Houten et al, Phys Rev B 37, 8534 (1988)

${ }^{81} \mathrm{D}$ A Wharam et al, J Phys C 21, L209(1988)

${ }^{82} \mathrm{P}$ C Man et al, Phys Rev B 40, 10033 (1989)

${ }^{83} \mathrm{C}$ W J Beenakker and $\mathrm{H}$ van Houten, Phys Rev B 39, 10445 (1989) 
${ }^{84}$ L. P. Kouwenhoven et al., Phys. Rev. B 40, 8083 (1989).

${ }^{85}$ B. J. van Wees et al., Phys. Rev. Lett. 62, 2523 (1989).

${ }^{86}$ Inter- and intra-Landau-level scattering in cross-gate devices has been studied by R. J. Haug, J. Kucera, P. Streda, and K. von Klitzing, Phys. Rev. B 39, 10892 (1989).

${ }^{87}$ Electron transport in the transition regions between the quantum Hall plateaus has been studied theoretically by $B$. Shapiro, Phys. Rev. B 33, 8447 (1986).

${ }^{88}$ B. E. Kane et al., Phys. Rev. Lett. 61, 1123 (1989); R. Woltjer, thesis, Utrecht, The Netherlands, 1988.

${ }^{89}$ Faist et al. (unpublished) have recently shown that interior bulk contacts can be used to preferentially populate the upper Landau level.

${ }^{90}$ The experiments by Komiyama et al. (Ref. 70) show that bulk contacts need not always be ideal. It may be that bulk contacts never achieve complete mixing of the edge channels, but the deviations from ideality may usually be too small to be detected. 